\title{
Design, synthesis and cytotoxic activity of some novel compounds containing pyrazolo[3,4- $d]$ pyrimidines nucleus
}

\author{
MANAL M KANDEEL ${ }^{\mathrm{a}}$, SAMEHA M ROSHDY ${ }^{\mathrm{a}}$, EMAN K A ABDELALL ${ }^{\mathrm{b}}$, \\ MOHAMED A ABDELGAWAD ${ }^{\mathrm{b}}$ and PHOEBE F LAMIE L $^{\mathrm{b}, *}$ \\ ${ }^{a}$ Pharmaceutical Organic Chemistry Department, Faculty of Pharmacy, Cairo University, Giza, Egypt \\ ${ }^{b}$ Pharmaceutical Organic Chemistry Department, Faculty of Pharmacy, Beni Suef University, Beni Suef, Egypt \\ e-mail: feby.farag@yahoo.com
}

MS received 10 August 2012; revised 10 March 2013; accepted 17 May 2013

\begin{abstract}
Novel pyrazolo[3,4- $d$ ]pyrimidines were designed and synthesized as antitumour agents against human breast cancer adenoma (MCF-7). Molecular modelling and pharmacological screening were performed against breast cancer cell line and also certain synthetic pathways were developed in order to introduce functionality onto C6 and N5 positions of pyrimidine moiety. Surprisingly, all the test compounds showed $\mathrm{IC}_{50}$ lower than that of the standard olomoucine I, especially compounds $\mathbf{4 b}, \mathbf{8 a}, \mathbf{1 0 b}, \mathbf{1 1 a}$ and $\mathbf{b}$, which showed $\mathrm{IC}_{50}$ between 0.009 and $0.004 \mu \mathrm{M}$.
\end{abstract}

Keywords. Pyrazolo[3,4- $d$ ] pyrimidine derivatives; cytotoxic activity; MCF-7; cyclin-dependent kinase inhibitors (CDKI).

\section{Introduction}

Cyclin-dependent kinases (CDK) are enzymes of Ser/Thr kinase type. This family of enzymes plays an important role in cell cycle regulation and proliferation. ${ }^{1} \mathrm{CDK}$ enzymatic regulation requires the binding of catalytic subunits (CDK1-CDK8) with regulatory subunits (cyclin A-cyclin $\mathrm{H}$ ) to give a fully active complex (CDK-cyclin), which is necessary for phosphorylation of key proteins that regulate the progression through the cell cycle. ${ }^{2}$

Several types of CDK inhibitors (staurosporine, flavopiridole, butyrolactone-1 and purine derivatives) have so far been described. ${ }^{2}$

Insight into different biological activities of pyrazolo $[3,4-d]$ pyrimidines as antimetabolites in purine biochemical reactions, were gained and several mechanisms were described for their cytotoxic activities as EGFR inhibitors, ${ }^{3}$ GSK-3 inhibitors, ${ }^{4,5}$ xanthine oxidase inhibitors, ${ }^{6}$ Mer receptor tyrosine kinase inhibitors, ${ }^{7}$ tyrosine kinase c-Src inhibitors, ${ }^{8-11}$ adenosine receptor antagonist ${ }^{12,13}$ and CDK inhibitors. ${ }^{14,15}$

CDKs are involved in the control of the cell cycle, but are overexpressed or overactive in many cancer cells. Since these enzymes are inactive in normal resting cells, drugs that target them should have fewer and less toxic side effects than conventional cytotoxic drugs, CDKs

*For correspondence are activated by cyclins via binding of a cyclin with its associated kinase, which activates the enzyme and serves to move the cell from one phase of the cell cycle to another, and inhibited by CDKIs. So, particular attention has been focused on CDKIs that offer selective and tolerable treatment for cancer. ${ }^{16}$

Moreover, pyrazolo [3, 4- $d$ ] pyrimidines were known to posses a broad spectrum of biological activities including antimicrobial ${ }^{17-19}$ antiviral $^{20,21}$ and antitumour activities. ${ }^{22-25}$

Herein, we have described the design, synthesis and pharmacological profile for a new class of compounds containing pyrazolo $[3,4-d]$ pyrimidine scaffold and having structure similar to olomoucine $\mathbf{I}$, the known potent CDK inhibitor. ${ }^{26}$

\section{Experimental}

\subsection{Docking studies}

Molecular modelling was performed using the X-ray crystal structure of olomoucine $\mathbf{I}$ bound to CDK using the 'Molecular Operating Environment (MOE) version 2008.10'.

Hydrogen atoms were added to amino acids of the protein and free energy calculation was assigned. Ligand for docking was prepared, atom types and protonation states and formal charges were assigned 
using default settings. The root mean square deviation (RMSD) of the docked conformation of olomoucine I was $0.52 \AA$ and the binding energy score was $-19.41 \mathrm{Kcal} / \mathrm{mol}$. Test compounds were prepared as above and the top 10 poses were retained for each molecule from every docking run.

\subsection{General}

Melting points were determined on a Graffin apparatus and were uncorrected. Element analyses $(\mathrm{C}, \mathrm{H}$, and N) were carried out on a Perkin-Elmer 2400 Analyzer (Perkin-Elmer, Norwalk, CT, USA) at the Micro analytical unit of Cairo University, Egypt. All compounds were $\delta$ within $\pm 0.4 \%$ of theoretical values. IR spectra were determined as $\mathrm{KBr}$ discs on Shimadzu IR 435 Spectrophotometer and values were represented in $\mathrm{cm}^{-1} \cdot{ }^{1} \mathrm{H}-\mathrm{NMR}$ spectra were carried out on a Bruker $300 \mathrm{MHz}$ NMR Spectrophotometer (Bruker, Munich, Germany) in Cairo University, Egypt, using DMSO- $d_{6}$ as a solvent, TMS as internal standard and chemical shifts were recorded in ppm on $\delta$ scale. Mass spectra were run on Hewlett Packard 5988 Spectrometer, Micro Analytical Center, Cairo University, Egypt. Progress of the reactions was monitored by TLC using TLC sheets precoated with UV fluorescent silica gel MERCK $60 \mathrm{~F}$ 254 that were visualized by UV lamp.

\subsection{6-Chloromethyl-3-methyl-1-phenyl-1, 5-dihydro-pyrazolo [3, 4-d] pyrimidin-4-one (3)}

A mixture of compound 1 (1.06 g, $4.9 \mathrm{mmol})$ and chloroacetyl chloride $(0.65 \mathrm{~g}, 5.8 \mathrm{mmol})$ was heated at $80^{\circ} \mathrm{C}$ for $6 \mathrm{~h}$. The mixture was cooled and neutralized with sodium carbonate solution (10\%). The precipitated solid was collected and crystallized from acetone as white crystals ( $69 \%$ yield); m.p.: $246-248^{\circ} \mathrm{C}$; IR ( $\mathrm{KBr}$, $\mathrm{cm}^{-1}$ ): 3276, 2934, 2822, 1675; ${ }^{1} \mathrm{H}-\mathrm{NMR}$ (DMSO- $d_{6}$ ) $\delta=2.50\left(\mathrm{~s}, 3 \mathrm{H}, \mathrm{CH}_{3}\right), 4.59\left(\mathrm{~s}, 2 \mathrm{H}, \mathrm{CH}_{2}\right), 7.34-7.38$ (t, 1H, C $4-\mathrm{H}), 7.39-7.56\left(\mathrm{t}, 2 \mathrm{H}, \mathrm{C}_{3}-\mathrm{H}, \mathrm{C}_{5}-\mathrm{H}\right), 8.00$ $8.03\left(\mathrm{~d}, 2 \mathrm{H}, \mathrm{C}_{2}-\mathrm{H}, \mathrm{C}_{6}-\mathrm{H}\right)$, and $12.61(\mathrm{~s}, 1 \mathrm{H}, \mathrm{NH}$, $\mathrm{D}_{2} \mathrm{O}$ exchangeable); $\mathrm{MS}(\mathrm{m} / \mathrm{z}): 274\left(\mathrm{M}^{+}, 85.80 \%\right)$, 77 (100\%); Anal. Found: C, 56.84\%; H, 4.04\%; N, $20.40 \% ; \mathrm{C}_{13} \mathrm{H}_{11} \mathrm{ClN}_{4} \mathrm{O}$ Calcd. C, $56.88 \% ; \mathrm{H}, 4.10 \% ; \mathrm{N}$, $20.37 \%$.

\subsection{General procedure for the synthesis of $\mathbf{4} \boldsymbol{a}-\boldsymbol{d}$}

A mixture of compound $3(0.52 \mathrm{~g}, 1.9 \mathrm{mmol})$, the appropriate primary aromatic amine $(10 \mathrm{mmol})$ and absolute ethanol $(20 \mathrm{ml})$ was heated under reflux for
$8 \mathrm{~h}$. The solid that formed on hot, was filtered and crystallized from dioxane as white crystals.

2.4a 3-Methyl-1-phenyl-6-phenylaminomethyl-1, 5-dihydropyrazolo [3, 4-d] pyrimidin-4-one $(\mathbf{4 a})$ : White colour solids; yield: 58\%; m.p. 223-225 $\mathrm{C}$; IR $\left(\mathrm{KBr}, \mathrm{cm}^{-1}\right)$ : 3428, 2930, 2871, 1685, ${ }^{1} \mathrm{H}-\mathrm{NMR}$ (DMSO- $\left.d_{6}\right) \delta=$ 2.49 (s, 3H, $\left.\mathrm{CH}_{3}\right), 4.30-4.32$ (d, 2H, $\left.\mathrm{CH}_{2}\right), 6.03-6.08$ (t, $1 \mathrm{H}, \mathrm{NH}, \mathrm{D}_{2} \mathrm{O}$ exchangeable), 6.57-6.59 (t, $1 \mathrm{H}, \mathrm{C}_{4^{\prime}}-$ $\mathrm{H}), 6.60-6.62\left(\mathrm{~d}, 2 \mathrm{H}, \mathrm{C}_{2^{\prime}}-\mathrm{H}, \mathrm{C}_{6^{\prime}}-\mathrm{H}\right), 7.08-7.13(\mathrm{t}, 2 \mathrm{H}$, $\left.\mathrm{C}_{3^{\prime}}-\mathrm{H}, \mathrm{C}_{5^{\prime}}-\mathrm{H}\right), 7.29-7.32\left(\mathrm{t}, 1 \mathrm{H}, \mathrm{C}_{4}-\mathrm{H}\right), 7.40-7.45(\mathrm{t}$, $\left.2 \mathrm{H}, \mathrm{C}_{3}-\mathrm{H}, \mathrm{C}_{5}-\mathrm{H}\right), 7.92-7.95\left(\mathrm{~d}, 2 \mathrm{H}, \mathrm{C}_{2}-\mathrm{H}, \mathrm{C}_{6}-\mathrm{H}\right)$, 12.15 (s, $1 \mathrm{H}, \mathrm{NH}, \mathrm{D}_{2} \mathrm{O}$ exchangeable); $\mathrm{MS}(\mathrm{m} / \mathrm{z}): 331$ $\left(\mathrm{M}^{+}, 58.24 \%\right), 234$ (100\%); Anal. Found: C, 68.87\%; $\mathrm{H}, 5.01 \%$; N, 21.09\%; $\mathrm{C}_{19} \mathrm{H}_{17} \mathrm{~N}_{5} \mathrm{O}$ Calcd. C, 68.87\%; $\mathrm{H}, 5.17 \%$; N, 21.13\%.

2.4b 3-Methyl-1-phenyl-6-(p-tolylphenyl) aminomethyl1, 5-dihydro-pyrazolo [3, 4-d] pyrimidin-4-one (4b): White colour solids; yield: $63 \%$; m.p. $217-219^{\circ} \mathrm{C}$; IR $\left(\mathrm{KBr}, \mathrm{cm}^{-1}\right): 3427,2999,2924,1684 ;{ }^{1} \mathrm{H}-\mathrm{NMR}$ $\left(\mathrm{DMSO}-d_{6}\right) \delta=2.13\left(\mathrm{~s}, 3 \mathrm{H}, \mathrm{CH}_{3}-\mathrm{ph}\right), 2.49(\mathrm{~s}, 3 \mathrm{H}$, $\left.\mathrm{CH}_{3}\right), 4.25-4.29\left(\mathrm{~d}, 2 \mathrm{H}, \mathrm{CH}_{2}\right), 5.84-5.86(\mathrm{t}, 1 \mathrm{H}, \mathrm{NH}$, $\mathrm{D}_{2} \mathrm{O}$ exchangeable), 6.56-6.60 $\left(\mathrm{d}, 2 \mathrm{H}, \mathrm{C}_{2^{\prime}}-\mathrm{H}, \mathrm{C}_{6^{\prime}}-\mathrm{H}\right)$, 6.89-6.93 (d, 2H, $\left.\mathrm{C}_{3^{\prime}}-\mathrm{H}, \mathrm{C}_{5^{\prime}}-\mathrm{H}\right), 7.28-7.34\left(\mathrm{t}, 1 \mathrm{H}, \mathrm{C}_{4}-\right.$ $\mathrm{H}), 7.41-7.44\left(\mathrm{t}, 2 \mathrm{H}, \mathrm{C}_{3}-\mathrm{H}, \mathrm{C}_{5}-\mathrm{H}\right), 7.93-7.97(\mathrm{~d}, 2 \mathrm{H}$, $\left.\mathrm{C}_{2}-\mathrm{H}, \mathrm{C}_{6}-\mathrm{H}\right), 12.10$ (s, 1H, NH, $\mathrm{D}_{2} \mathrm{O}$ exchangeable); MS ( $m / z): 345\left(\mathbf{M}^{+}, 79.33 \%\right), 120$ (100\%); Anal. Found: C, 69.58\%; H, 5.54\%; N, 20.32\%; $\mathrm{C}_{20} \mathrm{H}_{19} \mathrm{~N}_{5} \mathrm{O}$ Calcd. C, 69.55\%; H, 5.54\%; N, 20.28\%.

2.4c 3-Methyl-1-phenyl-6-(p-hydroxyphenyl) aminomethyl-1, 5-dihydro-pyrazolo [3, 4-d] pyrimidin-4-one (4c): White colour solids; yield: 60\%; m.p. 298$300^{\circ} \mathrm{C}$; IR $\left(\mathrm{KBr}, \mathrm{cm}^{-1}\right): 3439,3249,2979,2924,1675$; ${ }^{1} \mathrm{H}-\mathrm{NMR}\left(\mathrm{DMSO}-d_{6}\right) \delta=2.48\left(\mathrm{~s}, 3 \mathrm{H}, \mathrm{CH}_{3}\right), 4.21-4.22$ (d, 2H, $\left.\mathrm{CH}_{2}\right), 5.49-5.51\left(\mathrm{t}, 1 \mathrm{H}, \mathrm{NH}, \mathrm{D}_{2} \mathrm{O}\right.$ exchangeable), 6.51-6.52 (d, 2H, $\left.\mathrm{C}_{2^{\prime}}-\mathrm{H}, \mathrm{C}_{6^{\prime}}-\mathrm{H}\right), 6.55-6.58$ (d, $\left.2 \mathrm{H}, \mathrm{C}_{3^{\prime}}-\mathrm{H}, \mathrm{C}_{5^{\prime}}-\mathrm{H}\right), 7.29-7.34\left(\mathrm{t}, 1 \mathrm{H}, \mathrm{C}_{4}-\mathrm{H}\right), 7.44$ $7.49\left(\mathrm{t}, 2 \mathrm{H}, \mathrm{C}_{3}-\mathrm{H}, \mathrm{C}_{5}-\mathrm{H}\right), 7.95-7.98\left(\mathrm{~d}, 2 \mathrm{H}, \mathrm{C}_{2}-\mathrm{H}, \mathrm{C}_{6}-\right.$ $\mathrm{H}), 8.48\left(\mathrm{~s}, 1 \mathrm{H}, \mathrm{OH}, \mathrm{D}_{2} \mathrm{O}\right.$ exchangeable), $12.10(\mathrm{~s}$, $1 \mathrm{H}, \mathrm{NH}, \mathrm{D}_{2} \mathrm{O}$ exchangeable); $\mathrm{MS}(\mathrm{m} / \mathrm{z}): 347\left(\mathrm{M}^{+}\right.$, 39.30\%), 77 (100\%); Anal. Found: C, 65.57\%; H, $5.20 \% ; \mathrm{N}, 19.99 \% ; \mathrm{C}_{19} \mathrm{H}_{17} \mathrm{~N}_{5} \mathrm{O}_{2}$ Calcd. C, $65.69 \% ; \mathrm{H}$, $4.93 \%$; N, $20.16 \%$.

2.4d 3-Methyl-1-phenyl-6-(p-carboxyphenyl) aminomethyl-1, 5-dihydro-pyrazolo [3, 4-d] pyrimidin-4-one $(4 d)$ : White colour solids; yield: 66\%; m.p. 281$283^{\circ} \mathrm{C}$; IR $\left(\mathrm{KBr}, \mathrm{cm}^{-1}\right): 3404,3263,1664 ;{ }^{1} \mathrm{H}-\mathrm{NMR}$ $\left(\mathrm{DMSO}-d_{6}\right) \delta=2.49\left(\mathrm{~s}, 3 \mathrm{H}, \mathrm{CH}_{3}\right), 4.39-4.41(\mathrm{~d}, 2 \mathrm{H}$, 
$\left.\mathrm{CH}_{2}\right), 6.70-6.82\left(\mathrm{~d}, 2 \mathrm{H}, \mathrm{C}_{2^{\prime}}-\mathrm{H}, \mathrm{C}_{6^{\prime}}-\mathrm{H}\right), 6.81-6.83$ (t, $1 \mathrm{H}, \mathrm{NH}, \mathrm{D}_{2} \mathrm{O}$ exchangeable), 7.24-7.29 (d, 2H, $\mathrm{C}_{3^{\prime}}-$ $\left.\mathrm{H}, \mathrm{C}_{5^{\prime}}-\mathrm{H}\right), 7.32-7.37\left(\mathrm{t}, 1 \mathrm{H}, \mathrm{C}_{4}-\mathrm{H}\right), 7.70-7.73(\mathrm{t}, 2 \mathrm{H}$, $\left.\mathrm{C}_{3}-\mathrm{H}, \mathrm{C}_{5}-\mathrm{H}\right), 7.86-7.88\left(\mathrm{~d}, 2 \mathrm{H}, \mathrm{C}_{2}-\mathrm{H}, \mathrm{C}_{6}-\mathrm{H}\right), 12.05$ (s, 1H, NH, $\mathrm{D}_{2} \mathrm{O}$ exchangeable), $12.27(\mathrm{~s}, 1 \mathrm{H}, \mathrm{OH}$, $\mathrm{D}_{2} \mathrm{O}$ exchangeable); $\mathrm{MS}(\mathrm{m} / z): 375\left(\mathrm{M}^{+}, 92.70 \%\right)$, 77 (100\%); Anal. Found: C, 63.86\%; H, 4.51\%; N, $18.66 \% ; \mathrm{C}_{20} \mathrm{H}_{17} \mathrm{~N}_{5} \mathrm{O}_{3}$ Calcd. C, 63.99\%; H, 4.56\%; N, $18.66 \%$.

\subsection{General procedure for the synthesis of $5 \boldsymbol{a}-\boldsymbol{d}$}

To a solution of the appropriate compound $4 \mathbf{a}-\mathbf{d}$ $(0.6 \mathrm{mmol})$ in propanol $(10 \mathrm{ml})$, formaldehyde solution ( $3 \mathrm{ml}, 0.10 \mathrm{~mol}$ ) was added while stirring for $10 \mathrm{~min}$. The solution was stirred continuously for $2 \mathrm{~h}$. The formed precipitate was filtered and crystallized from propanol as white crystals.

2.5a 1.3-Methyl-1-phenyl-7-phenyl-1, 4, 5, 6, 7,8 hexahydropyrazolo $[3,4-d]$ imidazo $[3,4-a]$ pyrimidin-4one (5a): White colour solids; yield: 57\%; m.p. 246$248^{\circ} \mathrm{C}$; IR (KBr, cm ${ }^{-1}$ ): 2990-2857, 1687; ${ }^{1} \mathrm{H}-\mathrm{NMR}$ $\left(\mathrm{DMSO}-d_{6}\right) \delta=2.54\left(\mathrm{~s}, 3 \mathrm{H}, \mathrm{CH}_{3}\right), 4.70\left(\mathrm{~s}, 2 \mathrm{H}, \mathrm{C}-\mathrm{CH}_{2}-\right.$ $\mathrm{N}), 5.37\left(\mathrm{~s}, 2 \mathrm{H}, \mathrm{N}-\mathrm{CH}_{2}-\mathrm{N}\right)$ and 6.75-6.79 (t, $1 \mathrm{H}, \mathrm{C}_{4^{\prime}}-$ $\mathrm{H}), 6.82-6.84\left(\mathrm{~d}, 2 \mathrm{H}, \mathrm{C}_{2^{\prime}}-\mathrm{H}, \mathrm{C}_{6^{\prime}}-\mathrm{H}\right), 7.26-7.31(\mathrm{t}, 2 \mathrm{H}$, $\left.\mathrm{C}_{3^{\prime}}-\mathrm{H}, \mathrm{C}_{5^{\prime}}-\mathrm{H}\right), 7.34-7.40\left(\mathrm{t}, 1 \mathrm{H}, \mathrm{C}_{4}-\mathrm{H}\right), 7.52-7.57$ (t, $\left.2 \mathrm{H}, \mathrm{C}_{3}-\mathrm{H}, \mathrm{C}_{5}-\mathrm{H}\right), 7.99-8.03\left(\mathrm{~d}, 2 \mathrm{H}, \mathrm{C}_{2}-\mathrm{H}, \mathrm{C}_{6}-\mathrm{H}\right) ; \mathrm{MS}$ $(\mathrm{m} / z): 343\left(\mathrm{M}^{+}, 44.69 \%\right), 77$ (100\%); Anal. Found: C, $69.99 \%$; H, 4.71\%; N, 20.56\%; $\mathrm{C}_{20} \mathrm{H}_{17} \mathrm{~N}_{5} \mathrm{O}$ Calcd. C, $69.96 \% ; \mathrm{H}, 4.99 \%$; N, $20.40 \%$.

2.5b 3-Methyl-1-phenyl-7-(p-tolylphenyl)-1, 4, 5, 6, 7,8 hexahydropyrazolo [3, 4-d] imidazo [3, 4-a] pyrimidin-4-one $(\mathbf{5 b})$ : White colour solids; yield: $56 \%$; m.p. $284-286^{\circ} \mathrm{C}$; IR $\left(\mathrm{KBr}, \mathrm{cm}^{-1}\right)$ : 2921-2858, $1704 ;{ }^{1} \mathrm{H}-\mathrm{NMR}\left(\mathrm{DMSO}-d_{6}\right) \delta=2.22\left(\mathrm{~s}, 3 \mathrm{H}, \mathrm{CH}_{3}-\mathrm{ph}\right.$ ), 2.48(s, 3H, $\left.\mathrm{CH}_{3}\right), 4.66$ (s, 2H, C- $\left.\mathrm{CH}_{2}-\mathrm{N}\right), 5.34$ (s, $\left.2 \mathrm{H}, \mathrm{N}-\mathrm{CH}_{2}-\mathrm{N}\right)$ and 6.67-6.70 $\left(\mathrm{d}, 2 \mathrm{H}, \mathrm{C}_{2^{\prime}}-\mathrm{H}, \mathrm{C}_{6^{\prime}}-\right.$ $\mathrm{H}), 7.08-7.11\left(\mathrm{~d}, 2 \mathrm{H}, \mathrm{C}_{3^{\prime}}-\mathrm{H}, \mathrm{C}_{5^{\prime}}-\mathrm{H}\right), 7.30-7.37(\mathrm{t}$, $\left.1 \mathrm{H}, \mathrm{C}_{4}-\mathrm{H}\right), 7.52-7.58\left(\mathrm{t}, 2 \mathrm{H}, \mathrm{C}_{3}-\mathrm{H}, \mathrm{C}_{5}-\mathrm{H}\right), 7.99-8.02$ $\left(\mathrm{d}, 2 \mathrm{H}, \mathrm{C}_{2}-\mathrm{H}, \mathrm{C}_{6}-\mathrm{H}\right) ; \mathrm{MS}(\mathrm{m} / z): 357\left(\mathrm{M}^{+}, 63.96 \%\right)$, 240 (100\%); Anal. Found: C, 70.40\%; H, 5.49\%; N, $19.41 \% ; \mathrm{C}_{21} \mathrm{H}_{19} \mathrm{~N}_{5} \mathrm{O}$ Calcd. C, 70.57\%; H, 5.36\%; N, $19.59 \%$.

2.5c 3-Methyl-1-phenyl-7-(p-hydroxyphenyl)-1, 4,5,6,7, 8 hexahydropyrazolo[3,4-d]imidazo[3,4-a]pyrimidin4-one (5c): White colour solids; yield: 71\%; m.p. 253-255 $\mathrm{C}$; IR $\left(\mathrm{KBr}, \mathrm{cm}^{-1}\right)$ : 3267, 2927-2873, 1688; ${ }^{1} \mathrm{H}-\mathrm{NMR}\left(\mathrm{DMSO}-d_{6}\right) \delta=2.54\left(\mathrm{~s}, 3 \mathrm{H}, \mathrm{CH}_{3}\right), 4.60(\mathrm{~s}$, $\left.2 \mathrm{H}, \mathrm{C}-\mathrm{CH}_{2}-\mathrm{N}\right), 5.29\left(\mathrm{~s}, 2 \mathrm{H}, \mathrm{N}-\mathrm{CH}_{2}-\mathrm{N}\right), 6.66-6.72$ $\left(\mathrm{d}, 2 \mathrm{H}, \mathrm{C}_{2^{\prime}}-\mathrm{H}, \mathrm{C}_{6^{\prime}}-\mathrm{H}\right), 6.95-6.99\left(\mathrm{~d}, 2 \mathrm{H}, \mathrm{C}_{3^{\prime}}-\mathrm{H}, \mathrm{C}_{5^{\prime}}-\right.$ $\mathrm{H}), 7.32-7.41\left(\mathrm{t}, 1 \mathrm{H}, \mathrm{C}_{4}-\mathrm{H}\right), 7.52-7.60\left(\mathrm{t}, 2 \mathrm{H}, \mathrm{C}_{3}-\mathrm{H}\right.$, $\left.\mathrm{C}_{5}-\mathrm{H}\right), 7.99-8.02\left(\mathrm{~d}, 2 \mathrm{H}, \mathrm{C}_{2}-\mathrm{H}, \mathrm{C}_{6}-\mathrm{H}\right), 8.80(\mathrm{~s}, 1 \mathrm{H}$, $\mathrm{OH}, \mathrm{D}_{2} \mathrm{O}$ exchangeable); $\mathrm{MS}(\mathrm{m} / z): 359\left(\mathrm{M}^{+}, 0.27 \%\right)$, 240 (100\%); Anal. Found: C, 66.79\%; H, 4.59\%; N, 19.39\%; $\mathrm{C}_{20} \mathrm{H}_{17} \mathrm{~N}_{5} \mathrm{O}_{2}$ Calcd. C, 66.84\%; H, 4.77\%; N, $19.49 \%$.

2.5d 3-Methyl-1-phenyl-7-(carboxyphenyl)-1, 4, 5, 6, 7,8 hexahydropyrazolo [3, 4-d] imidazo [3, 4-a] pyrimidin-4-one $(\mathbf{5 d})$ : White colour solids; yield: 59\%; m.p. 346-348 ${ }^{\circ} \mathrm{C}$; IR ( $\left.\mathrm{KBr}, \mathrm{cm}^{-1}\right)$ : 3404, 1664; ${ }^{1} \mathrm{H}-\mathrm{NMR}\left(\mathrm{DMSO}-d_{6}\right) \delta=2.49\left(\mathrm{~s}, 3 \mathrm{H}, \mathrm{CH}_{3}\right), 4.39-$ $4.41\left(\mathrm{~d}, 2 \mathrm{H}, \mathrm{CH}_{2}\right), 6.70-6.82\left(\mathrm{~d}, 2 \mathrm{H}, \mathrm{C}_{2^{\prime}}-\mathrm{H}, \mathrm{C}_{6^{\prime}}-\right.$ $\mathrm{H})$, 6.81-6.83 (t, 1H, NH, $\mathrm{D}_{2} \mathrm{O}$ exchangeable), 7.24$7.29\left(\mathrm{~d}, 2 \mathrm{H}, \mathrm{C}_{3^{\prime}}-\mathrm{H}, \mathrm{C}_{5^{\prime}}-\mathrm{H}\right), 7.32-7.37\left(\mathrm{t}, 1 \mathrm{H}, \mathrm{C}_{4}-\right.$ $\mathrm{H}), 7.70-7.73\left(\mathrm{t}, 2 \mathrm{H}, \mathrm{C}_{3}-\mathrm{H}, \mathrm{C}_{5}-\mathrm{H}\right), 7.86-7.88(\mathrm{~d}, 2 \mathrm{H}$, $\left.\mathrm{C}_{2}-\mathrm{H}, \mathrm{C}_{6}-\mathrm{H}\right), 12.05$ (s, 1H, NH, $\mathrm{D}_{2} \mathrm{O}$ exchangeable), 12.27 (s, $1 \mathrm{H}, \mathrm{OH}, \mathrm{D}_{2} \mathrm{O}$ exchangeable); $\mathrm{MS}(\mathrm{m} / \mathrm{z}): 375$ $\left(\mathrm{M}^{+}, 92.70 \%\right), 77$ (100\%); Anal. Found: C, 65.24\%; H, 4.50\%; N, 18.20\%; $\mathrm{C}_{21} \mathrm{H}_{17} \mathrm{~N}_{5} \mathrm{O}_{3}$ Calcd. C, 65.11\%; $\mathrm{H}$, $4.42 \% ; \mathrm{N}, 18.08 \%$.

\subsection{General procedure for the synthesis of $6 \mathbf{a}-\boldsymbol{c}$}

A mixture of compound $3(2.75 \mathrm{~g}, 0.01 \mathrm{~mol})$ and the appropriate secondary amine $(0.02 \mathrm{~mol})$ in absolute ethanol $(20 \mathrm{~mL})$ was heated under reflux for $3 \mathrm{~h}$. The separated solid on hot, was filtered and crystallized from aqueous ethanol as off-white crystals.

2.6a 6-Diethylaminomethyl-3-methyl-1-phenyl-1, 5dihydro-pyrazolo [3,4-d]pyrimidin-4-one (6a): White off-colour crystals; yield: $47 \%$; m.p. $158-160^{\circ} \mathrm{C}$; IR $\left(\mathrm{KBr}, \mathrm{cm}^{-1}\right): 3424,2966-2864,1674 ;{ }^{1} \mathrm{H}-\mathrm{NMR}$ (DMSO- $\left.d_{6}\right) \delta=0.97-1.02\left(\mathrm{t}, 6 \mathrm{H}, J=7.2 \mathrm{~Hz}, 2 \mathrm{CH}_{2}-\right.$ $\mathrm{CH}_{3}$ ), 2.50 (s, 3H, $\mathrm{CH}_{3}$ ), 2.58-2.65 (q, $4 \mathrm{H}, J=7.2 \mathrm{~Hz}$, $\left.2 \mathrm{CH}_{2}-\mathrm{CH}_{3}\right), 3.60$ (s, $\left.2 \mathrm{H}, \mathrm{CH}_{2}\right), 7.31-7.36\left(\mathrm{t}, 1 \mathrm{H}, \mathrm{C}_{4}-\right.$ $\mathrm{H}), 7.45-7.54\left(\mathrm{t}, 2 \mathrm{H}, \mathrm{C}_{3}-\mathrm{H}, \mathrm{C}_{5}-\mathrm{H}\right), 8.02-8.06(\mathrm{~d}, 2 \mathrm{H}$, $\left.\mathrm{C}_{2}-\mathrm{H}, \mathrm{C}_{6}-\mathrm{H}\right)$, NH not visible; $\mathrm{MS}(\mathrm{m} / z): 311\left(\mathrm{M}^{+}\right.$, $0.34 \%), 72$ (100\%); Anal. Found: C, 65.85\%; H, 6.40\%; N, 22.51\%; $\mathrm{C}_{17} \mathrm{H}_{21} \mathrm{~N}_{5} \mathrm{O}$ Calcd. C, 65.57\%; H, 6.80\%; $\mathrm{N}, 22.49 \%$.

2.6b 3-Methyl-1-phenyl-6-piperidin-1-ylmethyl-1,5dihydro-pyrazolo [3,4-d]pyrimidin-4-one $(\boldsymbol{6} \boldsymbol{b})$ : White off-colour crystals; yield: 62\%; m.p. $195-197^{\circ} \mathrm{C}$; IR $\left(\mathrm{KBr}, \mathrm{cm}^{-1}\right): 3439,2995-2852,1673 ;{ }^{1} \mathrm{H}-\mathrm{NMR}$ $\left(\mathrm{DMSO}-d_{6}\right) \delta=1.59\left(\mathrm{~m}, 4 \mathrm{H}, 2 \mathrm{CH}_{2}\right), 1.83(\mathrm{~m}, 2 \mathrm{H}$, 
$\left.\mathrm{CH}_{2}\right), 2.64$ (s, 3H, $\left.\mathrm{CH}_{3}\right), 2.86\left(\mathrm{~m}, 4 \mathrm{H}, 2 \mathrm{~N}-\mathrm{CH}_{2}\right), 7.32-$ 7.35 (t, 1H, C $4-\mathrm{H}), 7.48-7.59\left(\mathrm{t}, 2 \mathrm{H}, \mathrm{C}_{3}-\mathrm{H}, \mathrm{C}_{5}-\mathrm{H}\right)$, 7.99-8.01 (d, 2H, $\left.\mathrm{C}_{2}-\mathrm{H}, \mathrm{C}_{6}-\mathrm{H}\right)$, NH not visible; $\mathrm{MS}$ $(\mathrm{m} / \mathrm{z}): 323\left(\mathrm{M}^{+}, 0.5 \%\right), 84(100 \%)$; Anal. Found: $\mathrm{C}$, $66.61 \% ; \mathrm{H}, 6.29 \% ; \mathrm{N}, 21.55 \% ; \mathrm{C}_{18} \mathrm{H}_{21} \mathrm{~N}_{5} \mathrm{O}$ Calcd. C, $66.85 \% ; \mathrm{H}, 6.55 \% ; \mathrm{N}, 21.66 \%$.

2.6c 3-Methyl-6-morpholin-4-ylmethyl-1-phenyl-1, 5dihydro-pyrazolo $[3,4-d]$ pyrimidin-4-one $(\boldsymbol{6 c})$ : White off-colour crystals; yield: $62 \%$; m.p. $219-221^{\circ} \mathrm{C}$; IR $\left(\mathrm{KBr}, \mathrm{cm}^{-1}\right): 3467,2990-2811,1670 ;{ }^{1} \mathrm{H}-\mathrm{NMR}$ $\left(\mathrm{DMSO}-d_{6}\right) \delta=2.48-2.59\left(\mathrm{~m}, 7 \mathrm{H}, \mathrm{CH}_{3}\right.$ and $2 \mathrm{CH}_{2}-$ O), $3.50\left(\mathrm{~s}, 2 \mathrm{H}, \mathrm{CH}_{2}\right), 3.58-3.61\left(\mathrm{~m}, 4 \mathrm{H}, 2 \mathrm{CH}_{2}-\mathrm{N}\right)$, 7.32-7.34 (t, 1H, $\left.\mathrm{C}_{4}-\mathrm{H}\right), 7.49-7.56\left(\mathrm{t}, 2 \mathrm{H}, \mathrm{C}_{3}-\mathrm{H}, \mathrm{C}_{5}-\right.$ $\mathrm{H})$, 8.01-8.05 (d, 2H, $\left.\mathrm{C}_{2}-\mathrm{H}, \mathrm{C}_{6}-\mathrm{H}\right)$; MS $(m / z): 325$ $\left(\mathrm{M}^{+}, 0.64 \%\right), 206$ (100\%); Anal. Found: C, 62.91\%; H, 5.66\%; N, 21.32\%; $\mathrm{C}_{17} \mathrm{H}_{19} \mathrm{~N}_{5} \mathrm{O}_{2}$ Calcd. C, 62.75\%; H, $5.89 \% ; \mathrm{N}, 21.52 \%$.

\subsection{General procedure for the synthesis of $7 \boldsymbol{a}-\boldsymbol{c}$}

A solution of compound $3(0.82 \mathrm{~g}, 3 \mathrm{mmol})$ in sodium (methoxide, ethoxide and/or propoxide) solution $(0.46 \mathrm{~g}$ sodium in $30 \mathrm{ml}$ absolute methanol, ethanol or propanol) was heated under reflux for $2 \mathrm{~h}$, the solvent was concentrated to half its volume and acidified with $(0.01 \mathrm{~N})$ hydrochloric acid until just acidic to litmus paper. The separated solid was filtered and crystallized from aqueous ethanol as white crystals.

2.7a 6-Methoxymethyl-3-methyl-1-phenyl-1,5-dihydropyrazolo [3,4-d]pyrimidin-4-one (7a): Yellow colour solid; yield; 46\%; m.p. $199-201^{\circ} \mathrm{C}$; IR $\left(\mathrm{KBr}, \mathrm{cm}^{-1}\right)$ : 3454, 2995-2928, 1676; ${ }^{1} \mathrm{H}-\mathrm{NMR}$ (DMSO- $d_{6}$ ) $\delta=$ $2.47\left(\mathrm{~s}, 3 \mathrm{H}, \mathrm{CH}_{3}\right), 3.38\left(\mathrm{~s}, 3 \mathrm{H}, \mathrm{OCH}_{3}\right), 4.37(\mathrm{~s}, 2 \mathrm{H}$, $\mathrm{CH}_{2}$ ), 7.33-7.38 (t, $\left.1 \mathrm{H}, \mathrm{C}_{4}-\mathrm{H}\right), 7.51-7.56\left(\mathrm{t}, 2 \mathrm{H}, \mathrm{C}_{3}-\right.$ $\left.\mathrm{H}, \mathrm{C}_{5}-\mathrm{H}\right), 8.01-8.05\left(\mathrm{~d}, 2 \mathrm{H}, \mathrm{C}_{2}-\mathrm{H}, \mathrm{C}_{6}-\mathrm{H}\right), 12.20$ (s, $1 \mathrm{H}, \mathrm{NH}, \mathrm{D}_{2} \mathrm{O}$ exchangeable); MS $(\mathrm{m} / \mathrm{z}): 270\left(\mathrm{M}^{+}\right.$, $85.33 \%), 240$ (100\%); Anal. Found: C, 61.99\%; H, 5.25\%; N, 20.94\%; $\mathrm{C}_{14} \mathrm{H}_{14} \mathrm{~N}_{4} \mathrm{O}_{2}$ Calcd. C, 62.21\%; H, $5.22 \% ; \mathrm{N}, 20.73 \%$.

2.7b 6-Ethoxymethyl-3-methyl-1-phenyl-1, 5-dihydropyrazolo [3, 4-d] pyrimidin-4-one $(7 \boldsymbol{b})$ : Yellow colour solid; yield: 58\%; m.p. $164-166^{\circ} \mathrm{C}$; IR $\left(\mathrm{KBr}, \mathrm{cm}^{-1}\right)$ : 3436, 2977-2929, 1677; ${ }^{1} \mathrm{H}-\mathrm{NMR}$ (DMSO- $\left.d_{6}\right) \delta=$ 1.14-1.19 (t, $\left.3 \mathrm{H}, \mathrm{CH}_{2} \mathrm{CH}_{3}\right), 2.49$ (s, 3H, $\mathrm{CH}_{3}$ ), 3.553.62 (q, $\left.2 \mathrm{H}, \mathrm{CH}_{2} \mathrm{CH}_{3}\right), 4.39$ (s, 3H, $\left.\mathrm{CH}_{2}\right), 7.33-7.36$ $\left(\mathrm{t}, 1 \mathrm{H}, \mathrm{C}_{4}-\mathrm{H}\right), 7.49-7.56\left(\mathrm{t}, 2 \mathrm{H}, \mathrm{C}_{3}-\mathrm{H}, \mathrm{C}_{5}-\mathrm{H}\right), 8.04-$ $8.06\left(\mathrm{~d}, 2 \mathrm{H}, \mathrm{C}_{2}-\mathrm{H}, \mathrm{C}_{6}-\mathrm{H}\right)$ and $\mathrm{NH}$ not visible; $\mathrm{MS}$ $(m / z): 284\left(\mathrm{M}^{+}, 36.66 \%\right), 240$ (100\%); Anal. Found:
C, 63.12\%; H, 5.48\%; N, 19.84\%; $\mathrm{C}_{15} \mathrm{H}_{16} \mathrm{~N}_{4} \mathrm{O}_{2}$ Calcd. C, $63.37 \%$; H, 5.67\%; N, $19.71 \%$.

2.7c 3-Methyl-1-phenyl-6-propoxymethyl-1, 5-dihydropyrazolo [3,4-d]pyrimidin-4-one $(7 \mathrm{c})$ : Yellow colour solid; yield: $30 \%$. m.p. $158-160^{\circ} \mathrm{C}$; IR $\left(\mathrm{KBr}, \mathrm{cm}^{-1}\right)$ : 3424, 2957-2871, 1686; ${ }^{1} \mathrm{H}-\mathrm{NMR}$ (DMSO- $d_{6}$ ) $\delta=$ 0.97-1.02 (t, 3H, $\left.\mathrm{CH}_{2}-\mathrm{CH}_{3}\right), 1.66-1.78\left(\mathrm{~m}, 2 \mathrm{H}, \mathrm{CH}_{2}-\right.$ $\left.\mathrm{CH}_{2}-\mathrm{CH}_{3}\right), 2.65\left(\mathrm{~s}, 3 \mathrm{H}, \mathrm{CH}_{3}\right), 3.57-3.62(\mathrm{t}, 2 \mathrm{H}, \mathrm{O}-$ $\mathrm{CH}_{2}-$ ), 4.53 (s, 2H, $\left.\mathrm{CH}_{2}-\mathrm{O}\right), 7.27-7.35\left(\mathrm{t}, 1 \mathrm{H}, \mathrm{C}_{4}-\mathrm{H}\right)$, 7.45-7.51 (t, 2H, $\left.\mathrm{C}_{3}-\mathrm{H}, \mathrm{C}_{5}-\mathrm{H}\right), 7.99-8.02$ (d, 2H, $\mathrm{C}_{2}-$ $\left.\mathrm{H}, \mathrm{C}_{6}-\mathrm{H}\right), 9.46\left(\mathrm{~s}, 1 \mathrm{H}, \mathrm{NH}, \mathrm{D}_{2} \mathrm{O}\right.$ exchangeable); $\mathrm{MS}$ $(\mathrm{m} / \mathrm{z}): 298\left(\mathrm{M}^{+}, 82.94 \%\right), 240(100 \%)$; Anal. Found: C, 64.20\%; H, 5.89\%; N, 18.37\%; $\mathrm{C}_{16} \mathrm{H}_{18} \mathrm{~N}_{4} \mathrm{O}_{2}$ Calcd. C, $64.41 \% ; \mathrm{H}, 6.08 \% ; \mathrm{N}, 18.78 \%$.

\subsection{General procedure for the synthesis of $8 \boldsymbol{a}$ and $\boldsymbol{b}$}

A mixture of compound $\mathbf{3}$ (2.75 g, $0.01 \mathrm{~mol})$, appropriate chloroacetanilide derivative $(0.01 \mathrm{~mol})$ and potassium hydroxide $(1.12 \mathrm{~g}, 0.02 \mathrm{~mol})$ in absolute ethanol $(25 \mathrm{ml})$ was heated under reflux for $24 \mathrm{~h}$. The separated solid on hot, was filtered, washed with water and crystallized from dioxane as off-white crystals.

2.8a 3-Methyl-1-phenyl-7oxo-8-phenyl-4, 5, 6, 7, 8, 9hexahydro-pyrazolo $[3,4-d]$ pyrazino $[4,3-a]$ pyrimidin4-one $(8 a)$ : White colour solid; yield: $18 \%$; m.p. 245$247^{\circ} \mathrm{C}$; IR $\left(\mathrm{KBr}, \mathrm{cm}^{-1}\right): 2916-2863,1704 ;{ }^{1} \mathrm{H}-\mathrm{NMR}$ $\left(\mathrm{DMSO}-d_{6}\right) \delta=2.54\left(\mathrm{~s}, 3 \mathrm{H}, \mathrm{CH}_{3}\right), 4.77\left(\mathrm{~s}, 2 \mathrm{H}, \mathrm{CH}_{2}-\right.$ $\mathrm{N}), 5.01\left(\mathrm{~s}, 2 \mathrm{H}, \mathrm{CH}_{2}-\mathrm{CO}\right), 7.27-8.04(\mathrm{~m}, 10 \mathrm{H}, \mathrm{Ar}-$ $\mathrm{H})$; MS $(\mathrm{m} / \mathrm{z})$ : $371\left(\mathbf{M}^{+}, 0.33 \%\right), 240(100 \%)$; Anal. Found: C, 67.80\%; H, 4.71\%; N, $18.70 \% ; \mathrm{C}_{21} \mathrm{H}_{17} \mathrm{~N}_{5} \mathrm{O}_{2}$ Calcd. C, 67.91\%; H, 4.61\%; N, $18.86 \%$.

$2.8 \mathrm{~b}$ 3-Methyl-1-phenyl-7oxo-8-(p-tolylphenyl)-4, 5,6,7,8,9-hexahydro-pyrazolo[3,4-d]pyrazino[4,3a]pyrimidin-4-one $(\boldsymbol{8 b})$ : White colour solid; yield: $15 \%$; m.p. $252-254^{\circ} \mathrm{C}$; IR $\left(\mathrm{KBr}, \mathrm{cm}^{-1}\right)$ : 2924-2850, 1705,$1583 ;{ }^{1} \mathrm{H}-\mathrm{NMR}$ (DMSO- $\left.d_{6}\right) \delta=2.22(\mathrm{~s}, 3 \mathrm{H}$, $\left.\mathrm{CH}_{3}\right), 2.49$ (s, 3H, $\left.\mathrm{CH}_{3}\right), 4.66$ (s, $\left.2 \mathrm{H}, \mathrm{CH}_{2}-\mathrm{N}\right), 5.34$ (s, $\left.2 \mathrm{H}, \mathrm{CH}_{2}-\mathrm{CO}\right), 6.67-6.70\left(\mathrm{~d}, 2 \mathrm{H}, \mathrm{C}_{2^{\prime}}-\mathrm{H}, \mathrm{C}_{6^{\prime}}-\mathrm{H}\right), 7.08$ $7.11\left(\mathrm{~d}, 2 \mathrm{H}, \mathrm{C}_{3^{\prime}}-\mathrm{H}, \mathrm{C}_{5^{\prime}}-\mathrm{H}\right), 7.35-7.40\left(\mathrm{t}, 1 \mathrm{H}, \mathrm{C}_{4}-\mathrm{H}\right)$, $7.52-7.58\left(\mathrm{t}, 2 \mathrm{H}, \mathrm{C}_{3}-\mathrm{H}, \mathrm{C}_{5}-\mathrm{H}\right), 7.99-8.02\left(\mathrm{~d}, 2 \mathrm{H}, \mathrm{C}_{2}-\right.$ $\left.\mathrm{H}, \mathrm{C}_{6}-\mathrm{H}\right) ; \mathrm{MS}(\mathrm{m} / \mathrm{z}): 385\left(\mathrm{M}^{+}, 55.56 \%\right), 206(100 \%)$; Anal. Found: C, 68.30\%; H, 4.91\%; N, 18.30\%; $\mathrm{C}_{22} \mathrm{H}_{19} \mathrm{~N}_{5} \mathrm{O}_{2}$ Calcd. C, 68.56\%; H, 4.97\%; N, $18.17 \%$. 
2.9 6-Mercaptomethyl-3-methyl-1-phenyl-1H-pyrazolo [3, 4-d] pyrimidin-4-one (9)

A mixture of compound $3(1.58 \mathrm{~g}, 5.75 \mathrm{mmol})$ and thiourea $(0.76 \mathrm{~g}, 0.01 \mathrm{~mol})$ in absolute ethanol $(25 \mathrm{ml})$ was heated under reflux for $4 \mathrm{~h}$. The product which was obtained on hot, was filtered, dissolved in sodium hydroxide $(20 \mathrm{ml}, 5 \%)$ and acidified with $(0.01 \mathrm{~N})$ hydrochloric acid until just acidic. The solid separated was collected and crystallized from dimethyl formamide as yellow colour crystals; yield: 50\%; m.p. $293-295^{\circ} \mathrm{C}$; IR $\left(\mathrm{KBr}, \mathrm{cm}^{-1}\right): 3426,2733,1675$; ${ }^{1} \mathrm{H}-\mathrm{NMR}\left(\mathrm{DMSO}-d_{6}\right) \quad \delta=2.48\left(\mathrm{~s}, 3 \mathrm{H}, \mathrm{CH}_{3}\right), 3.97$ $\left(\mathrm{s}, 2 \mathrm{H}, \mathrm{CH}_{2}\right), 7.28-7.31\left(\mathrm{t}, 1 \mathrm{H}, \mathrm{C}_{4}-\mathrm{H}\right), 7.42-7.47$ (t, $3 \mathrm{H}, \mathrm{C}_{3}-\mathrm{H}, \mathrm{C}_{5}-\mathrm{H}$ and $\mathrm{SH}\left(\mathrm{D}_{2} \mathrm{O}\right.$ exchangeable)), 7.99-8.01 (d, 2H, $\left.\mathrm{C}_{2}-\mathrm{H}, \mathrm{C}_{6}-\mathrm{H}\right), 12.35$ (s, 1H, NH, $\mathrm{D}_{2} \mathrm{O}$ exchangeable); $\mathrm{MS}(\mathrm{m} / \mathrm{z}): 272\left(\mathrm{M}^{+}, 45.30 \%\right)$, 198 (100\%); Anal. Found: C, 57.11\%; H, 4.55\%; N, $20.60 \% ; \mathrm{C}_{13} \mathrm{H}_{12} \mathrm{~N}_{4} \mathrm{OS}$ Calcd. C, $57.34 \% ; \mathrm{H}, 4.44 \%$; , $20.57 \%$.

Table 1. Docking study data and cytotoxic activity $\left(\mathrm{IC}_{50}\right)$ for olomoucine $\mathbf{I}$ and some newly synthesized compounds on CDK enzyme.

\begin{tabular}{|c|c|c|c|c|c|c|}
\hline $\begin{array}{l}\text { Compound } \\
\text { No. }\end{array}$ & $\begin{array}{l}\text { Number of } \\
\text { H-bonds }\end{array}$ & $\begin{array}{l}\text { Atoms of compound } \\
\text { forming H-bonds }\end{array}$ & $\begin{array}{l}\text { Amino acid residues } \\
\text { forming H-bonds }\end{array}$ & $\begin{array}{l}\text { Binding energy score } \\
(\mathrm{Kcal} / \mathrm{mol})\end{array}$ & $\begin{array}{c}\mathrm{IC}_{50} \\
(\mu \mathrm{g} / \mathrm{ml})\end{array}$ & $\begin{array}{l}\mathrm{IC}_{50} \\
(\mu \mathrm{M})\end{array}$ \\
\hline $4 b$ & & $\mathrm{C}=\mathrm{O}$, & Leu 83 & & & \\
\hline & 2 & $\mathrm{NH}$ & & -23.1725 & 2.7 & 0.008 \\
\hline $4 d$ & 3 & $\begin{array}{l}\mathrm{C}=\mathrm{O} \\
\mathrm{NH} \\
\mathrm{COO}^{-}\end{array}$ & $\begin{array}{l}\text { Leu } 83 \\
\text { Leu } 83 \\
\text { His } 84\end{array}$ & -17.6897 & 11.6 & 0.030 \\
\hline $5 \mathbf{a}$ & - & - & - & -23.8349 & 9.6 & 0.027 \\
\hline $5 c$ & 1 & $\mathrm{OH}$ & Lys 128 & -18.2302 & 8.84 & 0.013 \\
\hline $5 d$ & 2 & $\begin{array}{c}\mathrm{C}=\mathrm{O} \text { of }(\mathrm{COOH}), \\
\mathrm{C}-\mathrm{O}^{-}\end{array}$ & $\begin{array}{l}\text { Thr } 14 \\
\text { Lys } 129\end{array}$ & -11.7559 & 17.6 & 0.045 \\
\hline $6 a$ & 1 & $\mathrm{NH}$ & Asp 145 & -19.1579 & 5.55 & 0.017 \\
\hline $6 \mathbf{b}$ & 3 & $\begin{array}{c}\mathrm{C}=\mathrm{O} \\
\mathrm{NH} \\
\text { N piperidino group }\end{array}$ & $\begin{array}{l}\text { Leu } 83 \\
\text { Leu } 83 \\
\text { Lys } 89\end{array}$ & -23.0477 & 4.2 & 0.012 \\
\hline $6 c$ & 3 & $\begin{array}{c}\mathrm{C}=\mathrm{O}, \\
\mathrm{NH}, \\
\mathrm{N} \text { morpholino group }\end{array}$ & $\begin{array}{l}\text { Leu } 83 \\
\text { Leu } 83 \\
\text { Lys } 89\end{array}$ & -23.2674 & 17.7 & 0.054 \\
\hline $7 a$ & 1 & $\mathrm{C}=\mathrm{O}$ & Gln 131 & -18.1891 & 5.85 & 0.021 \\
\hline $7 b$ & 2 & $\begin{array}{c}\mathrm{C}=\mathrm{O} \\
\mathrm{NH}\end{array}$ & $\begin{array}{l}\text { Leu } 83 \\
\text { Leu } 83\end{array}$ & -21.2642 & 10.2 & 0.035 \\
\hline $7 c$ & 2 & $\begin{array}{l}\mathrm{C}=\mathrm{O}, \\
\mathrm{NH}\end{array}$ & $\begin{array}{l}\text { Leu } 83 \\
\text { Leu } 83\end{array}$ & -20.1295 & 13.2 & 0.044 \\
\hline $8 a$ & 1 & $\mathrm{C}=\mathrm{O}$ & Gln 131 & -24.2298 & 3.45 & 0.009 \\
\hline $8 b$ & 1 & $\mathrm{C}=\mathrm{O}$ & Gln 131 & -25.0835 & 6.6 & 0.080 \\
\hline $10 a$ & 2 & $\begin{array}{l}\mathrm{C}=\mathrm{O}, \\
\mathrm{NH}\end{array}$ & Leu 83 & -21.0402 & 8.4 & 0.023 \\
\hline $10 \mathrm{~b}$ & 2 & $\mathrm{C}=\mathrm{O}$, & $\begin{array}{l}\text { Leu } 83 \\
\text { Leu } 83\end{array}$ & -24.4210 & 2.85 & 0.007 \\
\hline $\begin{array}{l}11 \mathrm{a} \\
11 \mathrm{~b}\end{array}$ & 1 & $\begin{array}{l}\mathrm{C}=\mathrm{O} \\
\mathrm{C}=\mathrm{O}\end{array}$ & $\begin{array}{l}\text { Lys } 89 \\
\text { His } 84\end{array}$ & -16.2786 & 4.2 & 0.007 \\
\hline & 2 & $\begin{array}{c}\mathrm{C}=\mathrm{O}, \\
\mathrm{NH} \text { of }\left(-\mathrm{SCH}_{2} \mathrm{CONH}-\right)\end{array}$ & $\begin{array}{l}\text { His } 84 \\
\text { Asp } 56\end{array}$ & -21.6464 & 2.85 & 0.004 \\
\hline Olomoucine & 2 & $\begin{array}{l}\text { N6, } \\
\text { NH }\end{array}$ & $\begin{array}{l}\text { Leu } 83, \\
\text { Leu } 83\end{array}$ & -19.4146 & - & 7 \\
\hline
\end{tabular}

Bold numbers refer to the most potent compounds (lower IC50) relative to the standard 
2.10 General procedure for the synthesis of $10 \boldsymbol{a}$ and $\boldsymbol{b}$

A mixture of compound $9(1.04 \mathrm{~g}, 3.87 \mathrm{mmol})$, the appropriate halo compound $(3.87 \mathrm{mmol})$ and sodium acetate $(0.7 \mathrm{~g}, 8.50 \mathrm{mmol})$ in absolute ethanol was heated under reflux for $5 \mathrm{~h}$, and then allowed to cool. The separated solid was filtered, washed with water and crystallized from acetone as yellow crystals.

2.10a (3-Methyl-4-oxo-1-phenyl-4, 5-dihydro-1H-pyrazolo [3, 4-d] pyrimidin-6-ylmethylsulphanyl)-acetic acid ethyl ester (10a): Yellow colour solid; yield: 60\%; m.p. $160-162^{\circ} \mathrm{C}$; IR $\left(\mathrm{KBr}, \mathrm{cm}^{-1}\right)$ : 3445, 2982-2851, 1734, 1674; ${ }^{1} \mathrm{H}-\mathrm{NMR}$ (DMSO- $d_{6}$ ) $\delta=1.02-1.06$ (t, $\left.3 \mathrm{H}, \mathrm{CH}_{2} \mathrm{CH}_{3}\right), 2.49$ (s, $\left.3 \mathrm{H}, \mathrm{CH}_{3}\right), 3.54\left(\mathrm{~s}, 2 \mathrm{H},-\mathrm{CH}_{2}-\right.$ $\mathrm{S}), 3.73$ (s, 2H, S- $\mathrm{CH}_{2}$ ), 3.88-3.95 (q, $2 \mathrm{H}, \mathrm{CH}_{2} \mathrm{CH}_{3}$ ), 7.34-7.37 (t, 1H, $\left.\mathrm{C}_{4}-\mathrm{H}\right), 7.49-7.54\left(\mathrm{t}, 2 \mathrm{H}, \mathrm{C}_{3}-\mathrm{H}, \mathrm{C}_{5}-\right.$ $\mathrm{H}), 8.03-8.06\left(\mathrm{~d}, 2 \mathrm{H}, \mathrm{C}_{2}-\mathrm{H}, \mathrm{C}_{6}-\mathrm{H}\right), 12.33$ (s, $1 \mathrm{H}, \mathrm{NH}$, $\mathrm{D}_{2} \mathrm{O}$ exchangeable); $\mathrm{MS}(\mathrm{m} / \mathrm{z}): 358\left(\mathrm{M}^{+}, 17.90 \%\right)$, 240 (100\%). Anal. Found: C, 57.19\%; H, 5.25\%; N, $15.89 \% ; \mathrm{C}_{17} \mathrm{H}_{18} \mathrm{~N}_{4} \mathrm{O}_{3} \mathrm{~S}$ Calcd. C, 56.97\%; H, 5.06\%; $\mathrm{N}, 15.63 \%$.

2.10b 6-Benzylsulphanylmethyl-3-methyl-1-phenyl-1, 5-dihydro-pyrazolo[3,4-d]pyrimidin-4-one $(10 b)$ : Yellow colour solid; yield: $65 \%$; m.p. $217-219^{\circ} \mathrm{C}$; IR (KBr, $\mathrm{cm}^{-1}$ ): 3439, 2990-2857, 1687; ${ }^{1} \mathrm{H}-\mathrm{NMR}$ (DMSO-d6)

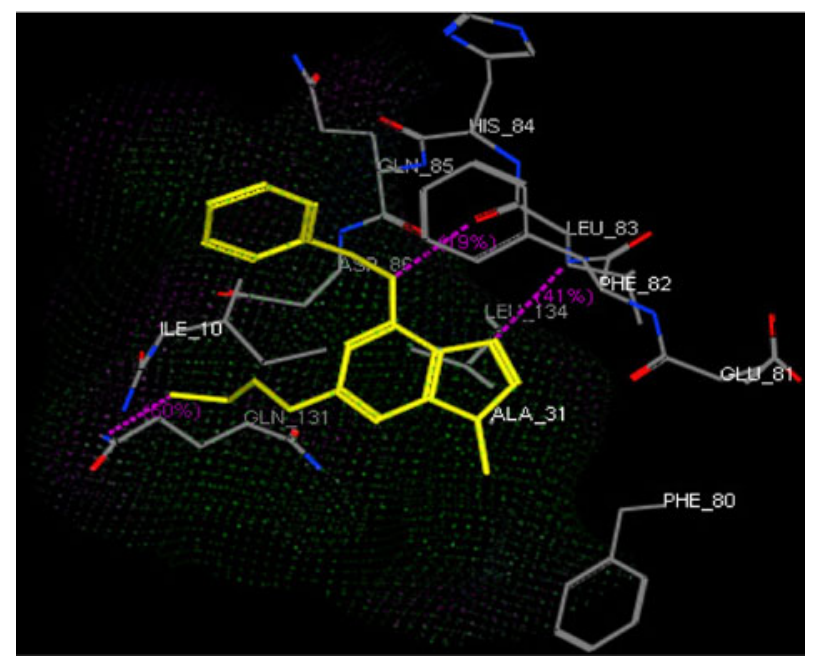

(a)

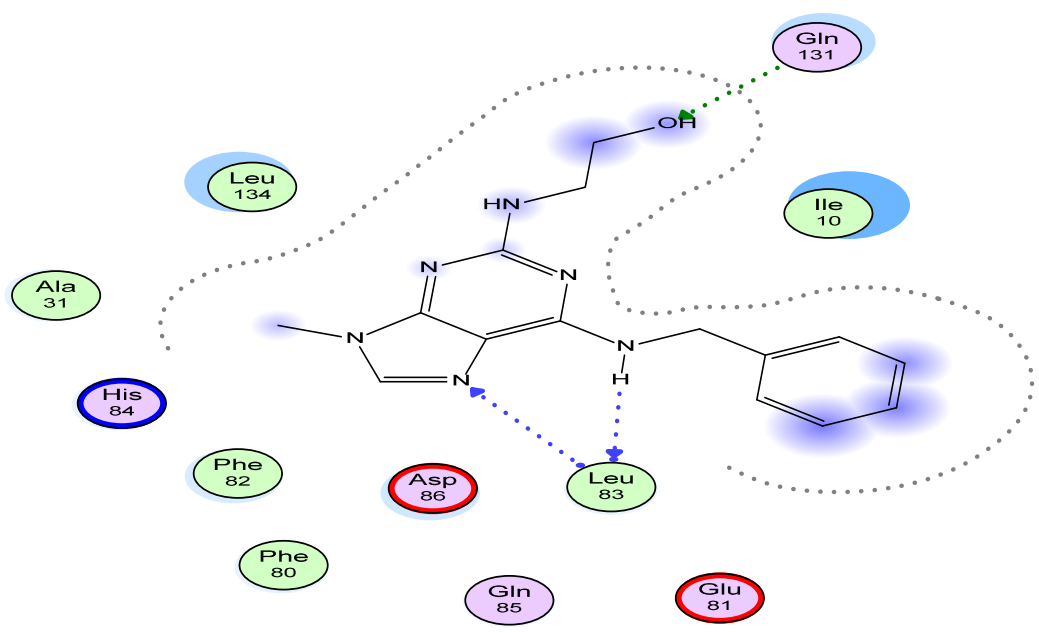

(b)

Figure 1. (a) Proposed binding mode of olomoucine I inside the active site of CDK resulting from docking, the most important amino acids are shown together with their respective numbers. It forms two $\mathrm{H}$-bonds with Leu 83 through its pyrimidine $\mathrm{C} 6-\mathrm{NH}$ and $\mathrm{N} 7$ of imidazol ring, and one H-bond with $\mathrm{C} 2$ side chain-OH group. (b) $2 \mathrm{D}$ interaction of olomoucine $\mathbf{I}$ with Leu 83 and Gln 131 amino acid. 


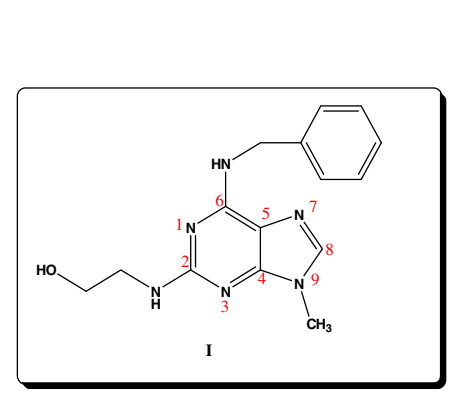

(a)

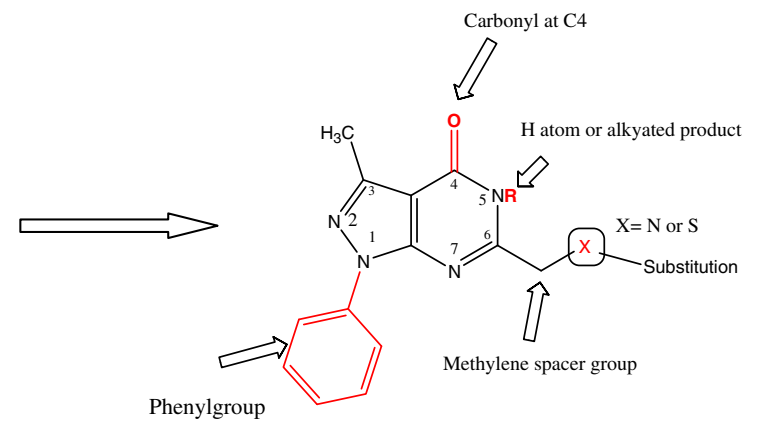

(b) II

Figure 2. (a) Structure of the lead compound olomoucine. (b) Common features for the newly synthesized compounds.

$\delta=2.38\left(\mathrm{~s}, 3 \mathrm{H}, \mathrm{CH}_{3}\right), 3.53\left(\mathrm{~s}, 2 \mathrm{H},-\mathrm{CH}_{2}-\mathrm{S}\right), 3.97(\mathrm{~s}$, $2 \mathrm{H}, \mathrm{S}-\mathrm{CH}_{2}-\mathrm{Ph}$ ), 7.18-8.09 (m, 10H, Ar-H), 12.31 (s, $1 \mathrm{H}, \mathrm{NH}, \mathrm{D}_{2} \mathrm{O}$ exchangeable); $\mathrm{MS}(\mathrm{m} / z): 362\left(\mathrm{M}^{+}\right.$, 21.10\%), 91 (100\%). Anal. Found: C, 66.20\%; H, $5.05 \% ; \mathrm{N}, 15.32 \% ; \mathrm{C}_{20} \mathrm{H}_{18} \mathrm{~N}_{4} \mathrm{OS}$ Calcd. C, 66.28\%; H, $5.01 \% ; \mathrm{N}, 15.46 \%$.

\subsection{General procedure for synthesis of $11 \boldsymbol{a}$ and $\boldsymbol{b}$}

A mixture of compound 9 (2.72 g, $0.01 \mathrm{~mol})$, appropriate anilide compound $(0.02 \mathrm{~mol})$ and potassium carbonate $(2.76 \mathrm{~g}, 0.02 \mathrm{~mol})$ in acetone $(20 \mathrm{ml})$, was heated under reflux for $10 \mathrm{~h}$, then allowed to cool.

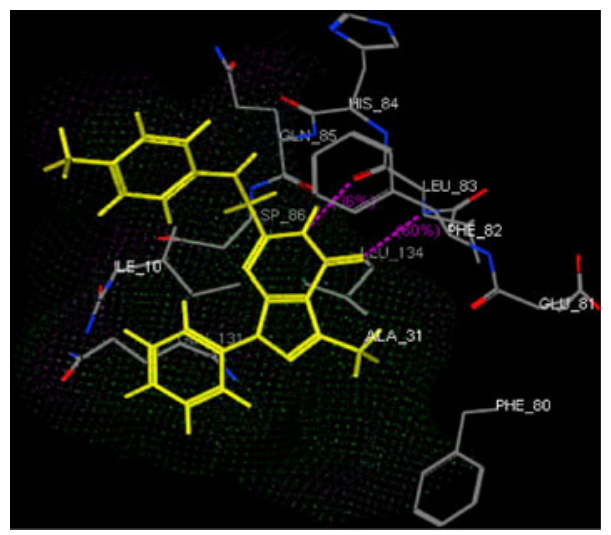

(a)

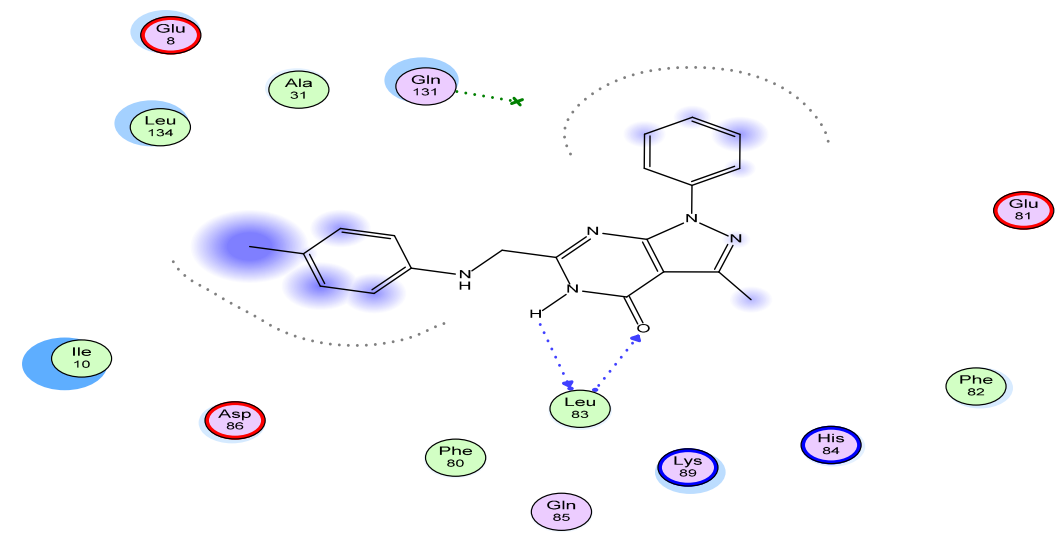

(b)

Figure 3. (a) Proposed binding mode of compound $\mathbf{4 b}$ inside the active site of CDK resulting from docking, the most important amino acids are shown together with their respective numbers. Compound $\mathbf{4 b}$ forms two $\mathrm{H}$-bonds with Leu 83 through its pyrimidine $\mathrm{NH}$ and carbonyl group. (b) 2D interaction of compound $\mathbf{7 b}$ with Leu 83 amino acid. 
The separated solid was filtered and crystallized from dioxane as yellow crystals.

2.11a 2-(3-Methyl-4-oxo-1-phenyl-5-phenylcarbamoyl methyl-4, 5-dihydro-1H-pyrazolo [3, 4-d] pyrimidin-6ylmethylsulphanyl)-N-phenyl-acetamide (11a): Yellow colour solid; yield: $58 \%$; m.p. $280-282^{\circ} \mathrm{C}$; IR ( $\mathrm{KBr}$, $\left.\mathrm{cm}^{-1}\right): 3437-289,2978.52-926,1691,1662 ;{ }^{1} \mathrm{H}-\mathrm{NMR}$ $\left(\mathrm{DMSO}-d_{6}\right) \delta=2.49$ (s, $\left.3 \mathrm{H}, \mathrm{CH}_{3}\right), 3.54\left(\mathrm{~s}, 2 \mathrm{H},-\mathrm{H}_{2}-\mathrm{S}\right)$, 4.09 (s, 2H, S- $\mathrm{CH}_{2}$ ), 5.08 (s, 2H, N-CH $-\mathrm{CO}$ ), 6.99$8.03(\mathrm{~m}, 15 \mathrm{H}, \mathrm{Ar}-\mathrm{H}), 9.94\left(\mathrm{~s}, 1 \mathrm{H}, \mathrm{NH}, \mathrm{D}_{2} \mathrm{O}\right.$ exchangeable), $10.45\left(\mathrm{~s}, 1 \mathrm{H}, \mathrm{NH}, \mathrm{D}_{2} \mathrm{O}\right.$ exchangeable); MS $(\mathrm{m} / \mathrm{z}): 538\left(\mathbf{M}^{+}, 20.26 \%\right), 281(100 \%)$; Anal. Found: C, 64.59\%; H, 4.29\%; N, 16.62\%; $\mathrm{C}_{29} \mathrm{H}_{26} \mathrm{~N}_{6} \mathrm{O}_{3} \mathrm{~S}$ Calcd. C, 64.67\%; H, 4.87\%; N, $15.60 \%$.

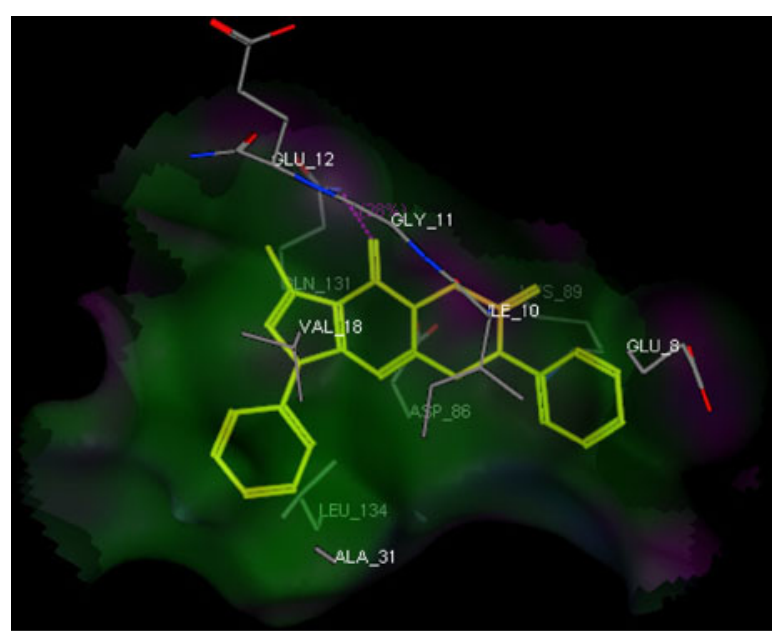

(a)

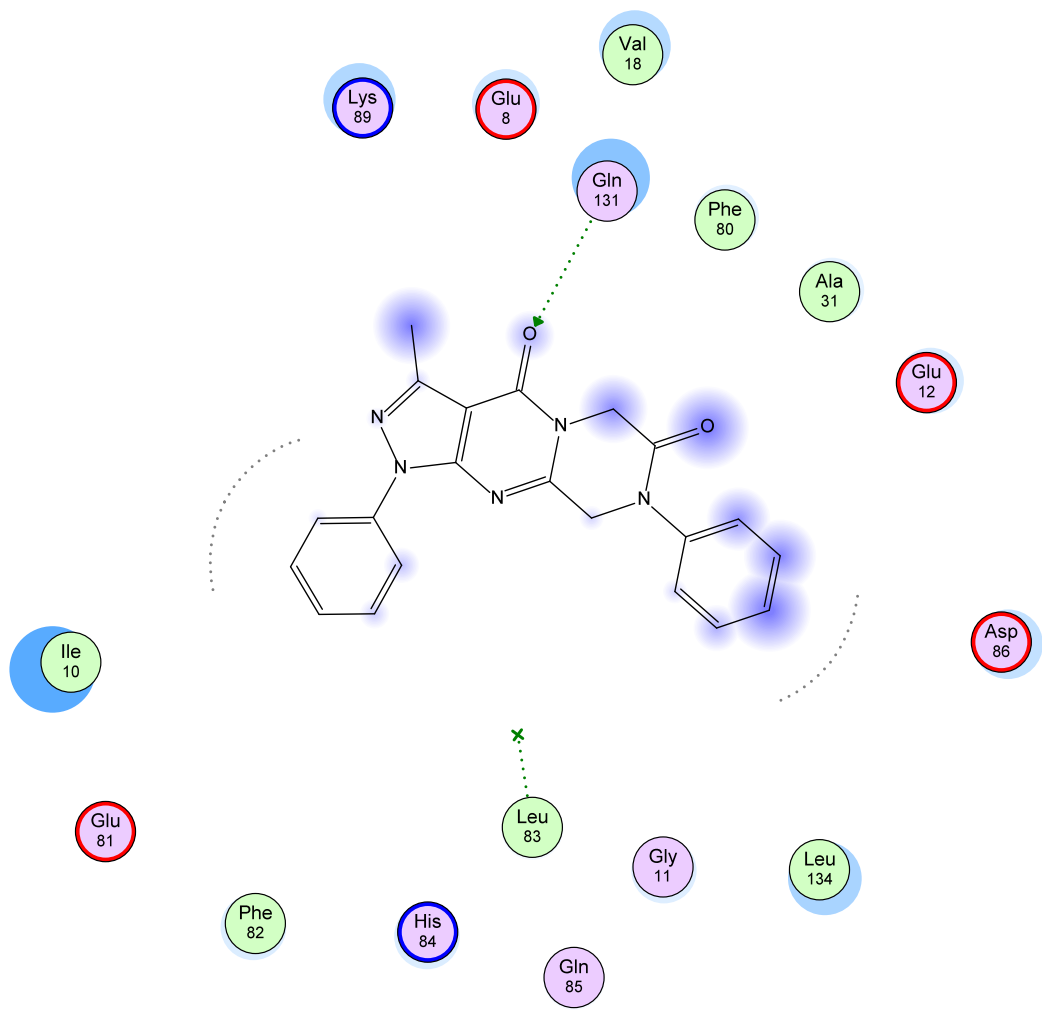

(b)

Figure 4. (a) Proposed binding mode of compound 8a inside the active site of CDK resulting from docking, the most important amino acids are shown together with their respective numbers. Compound 8a forms a H-bonds with Gln 131 through its pyrimidine $\mathrm{C}=\mathrm{O}$. (b) $2 \mathrm{D}$ interaction of compound 8a with Gln 131 amino acid. 
2.11b 2-[3-Methyl-4-oxo-1-phenyl-5-(p-tolylcarbamoylmethyl)-4, 5-dihydro-1H-pyrazolo [3, 4-d] pyrimidin6-ylmethylsulphanyl]-N-p-tolyl-acetamide

$(11 b)$ :

White colour solid; yield: $69 \%$. m.p.; $289-291{ }^{\circ} \mathrm{C}$; IR $\left(\mathrm{KBr}, \mathrm{cm}^{-1}\right)$ 3437-3304, 3035-2921, $1671 \quad(3 \mathrm{C}=\mathrm{O})$; ${ }^{1} \mathrm{H}-\mathrm{NMR}\left(\mathrm{DMSO}-d_{6}\right) \delta=2.19\left(\mathrm{~s}, 6 \mathrm{H}, 2 \mathrm{CH}_{3}\right), 2.49$ (s, $\left.3 \mathrm{H}, \mathrm{CH}_{3}\right), 3.49$ (s, 2H, $\left.-\mathrm{CH}_{2}-\mathrm{S}\right), 4.08\left(\mathrm{~s}, 2 \mathrm{H}, \mathrm{S}-\mathrm{CH}_{2}\right)$, 5.05 (s, 2H, N- $\left.\mathrm{CH}_{2}-\mathrm{CO}\right), 6.97-8.15(\mathrm{~m}, 13 \mathrm{H}, \mathrm{Ar}-\mathrm{H})$, $9.86\left(\mathrm{~s}, 1 \mathrm{H}, \mathrm{NH}, \mathrm{D}_{2} \mathrm{O}\right.$ exchangeable) and $10.36(\mathrm{~s}$, $1 \mathrm{H}, \mathrm{NH}, \mathrm{D}_{2} \mathrm{O}$ exchangeable); $\mathrm{MS}(\mathrm{m} / \mathrm{z}): 566\left(\mathrm{M}^{+}\right.$, $10.40 \%), 55$ (100\%); Anal. Found: C, 65.44\%; H, 5.56 $\% ; \mathrm{N}, 14.62 \% ; \mathrm{C}_{31} \mathrm{H}_{30} \mathrm{~N}_{6} \mathrm{O}_{3} \mathrm{~S}$ Calcd. C, 65.70\%; H, $5.34 \% ; \mathrm{N}, 14.83 \%$.

\subsection{Antitumour bioassay}

The effects of compounds on the growth of tumour cell lines (MCF-7), were evaluated according to the procedure adopted by the National Cancer Institute, Cairo, Egypt for the in vitro anticancer drug screening that use the protein-binding dye sulphorhodamine B (SRB) to assess growth inhibition. ${ }^{27}$ Cell were routinely maintained as adherent cell cultures in RPMI-1640 medium supplemented with $10 \%$ heat-inactived fetal bovine serum (FBS) and $1 \%$ penicillin/streptomycin at $37^{\circ} \mathrm{C}$ in humidified atmosphere containing $5 \% \mathrm{CO}_{2}$.

The cell line was regularly subcultured to be maintained in the exponential growth phase. Cells were exposed for $48 \mathrm{~h}$ to different concentrations (2.5$50 \mu \mathrm{g} / \mathrm{ml}$ ) of test compounds. Test compounds were prepared in dimethylsulphoxide (DMSO), were freshly diluted with cell culture medium just prior to the assays. Olomoucine I was used as positive control. For each test compound and cell line, a dose-response curve was generated and growth inhibition of $50 \%$ ( $\left.\mathrm{IC}_{50}\right)$, corresponding to the concentration of compound that inhibits

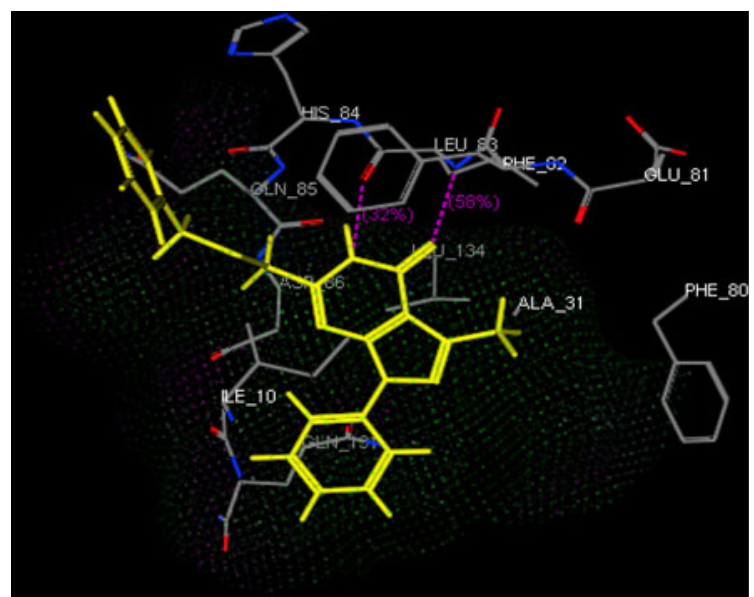

(a)

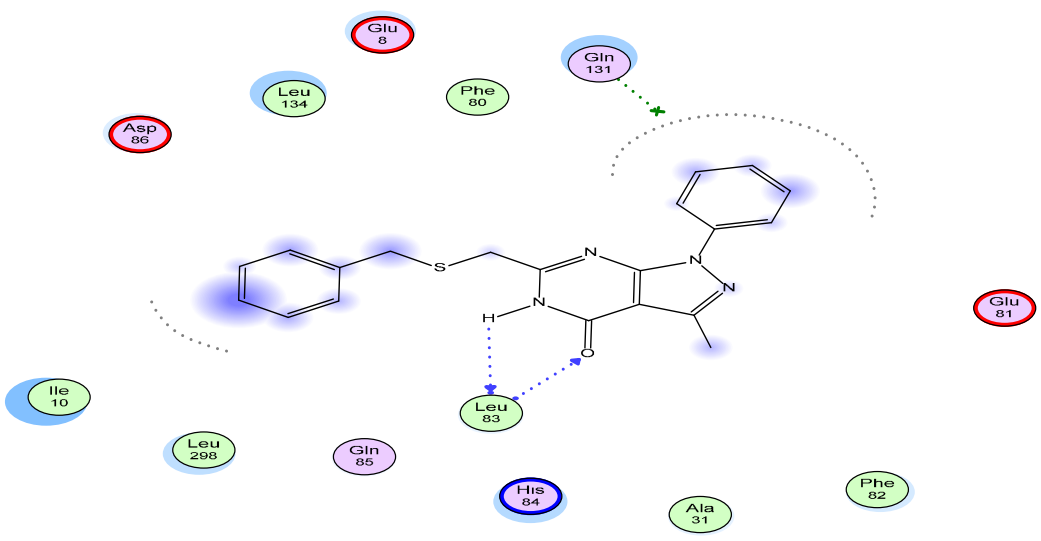

(b)

Figure 5. (a) The proposed binding mode of compound 10b inside the active site of CDK resulting from docking, the most important amino acids are shown together with their respective numbers. Compound $\mathbf{1 0 b}$ forms two H-bonds with Leu 83 through its pyrimidine $\mathrm{NH}$ and carbonyl group. (b) $2 \mathrm{D}$ interaction of compound $\mathbf{1 3 b}$ with Leu 83 amino acid. 
$50 \%$ of the net cell growth was determined. Results of in vitro cytotoxic activity experiments are presented in table 1.

\section{Results and discussion}

\subsection{Molecular modelling}

The lead compound olomoucine I (PDB ID: 1W0X), ${ }^{26}$ which was reported to have CDK inhibitory activity, was used to generate common feature hypothesis of CDK inhibitor as antitumour agents by introducing pyrazole ring instead of imidazole ring on the purine analogue. Both $\mathrm{N} 7$ and $\mathrm{NH}$ on $\mathrm{C} 6$ of imidazole ring of olomoucine I was found to form a H-bond with Leu 83 which is important for the activity. The $\mathrm{C} 2$ side chain is bound within the ATP ribose binding pocket, with the methyl group which interacts hydrophobically with glycine-rich loop and val 18 . The hydroxyl group of the side chain can form a H-bond with the backbone carbonyl of Gln 131. Purine N9 of methyl group shows a

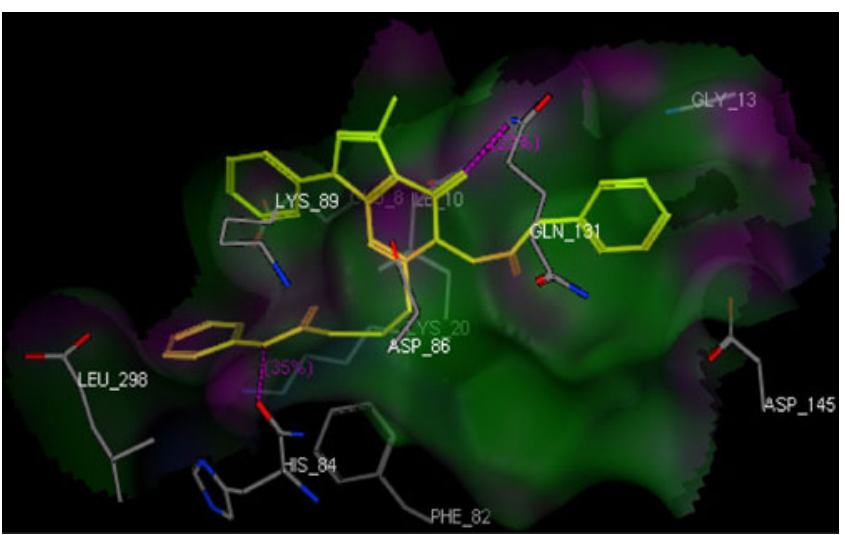

(a)

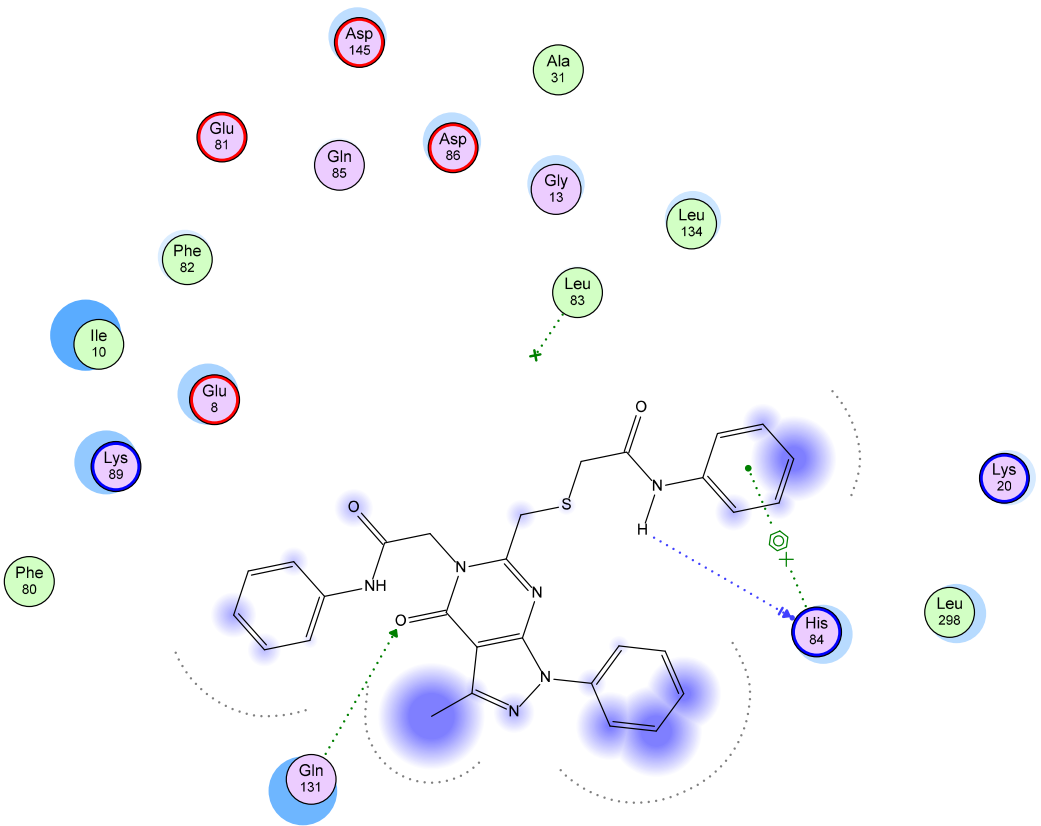

(b)

Figure 6. (a) Proposed binding mode of compound 11a inside the active site of CDK resulting from docking, the most important amino acids are shown together with their respective numbers. Compound 11a forms two H-bonds with His 84 through its NH group and with Gln 131 through its pyrimidine $\mathrm{C}=\mathrm{O}$ group, the other interaction is arene cation interaction with His 84 amino acid. (b) 2D interaction of compound 11a with His 84 and Gln 131 amino acids. 
strong hydrophobic interaction with hydrophobic side chains of Val 18, Ala 31, Phe 80, Leu 134 and Ala 144 (figure 1). ${ }^{1}$

Using the common features of the lead compound I, we designed new pyrazolo[3,4- $d]$ pyrimidine derivatives with the following modifications:

N1 Pyrazole nitrogen of pyrazolopyrimidine ring was substituted with phenyl group instead of the methyl group at N9 of the lead compound I. At the C6 side chain, we added additional carbon before $\mathrm{NH}$ group to act as a spacer that showed good fitting to the receptor. We explored the usage of alkyl or aromatic side chain at C6 in order to increase hydrophobic interaction towards the receptor. Additional modification was added at N5 of the pyrimidine ring to certain new compounds aiming to increase hydrogen bonding interaction with the receptor binding site (figure 2 ).

These modifications and docking of new compounds with CDK enzyme has given rise to a new common feature hypothesis for our compounds (figure $2 \mathrm{~b}$ ). This hypothesis showed that carbonyl group at $\mathrm{C} 4$ and $\mathrm{N} 5 \mathrm{H}$ from a hydrogen bond with Leu 83 and similar interaction was also stated for the lead compound I. Alteration of methyl group in I with phenyl group in II resulted in better hydrophobic interaction, hence phenyl is more deeply socked into ATP ribose binding pocket. By induction of different pharmacophores to C6 side chain, a variety of hydrogen bonding was estimated in

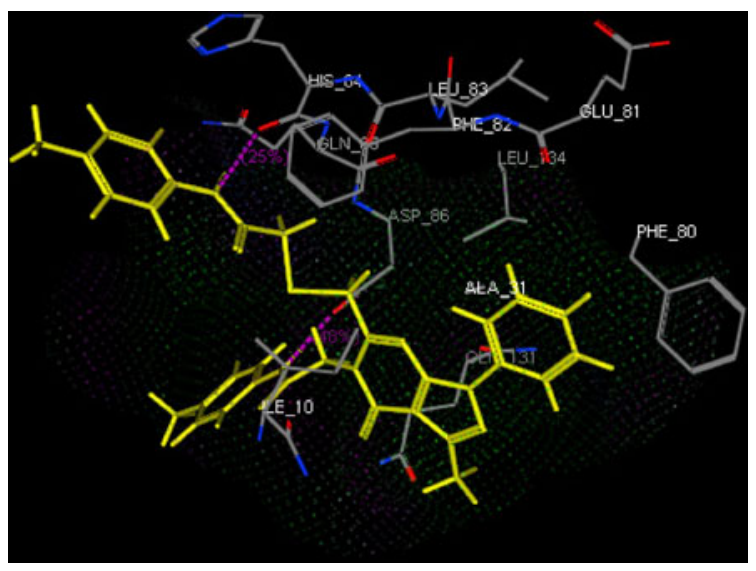

(a)

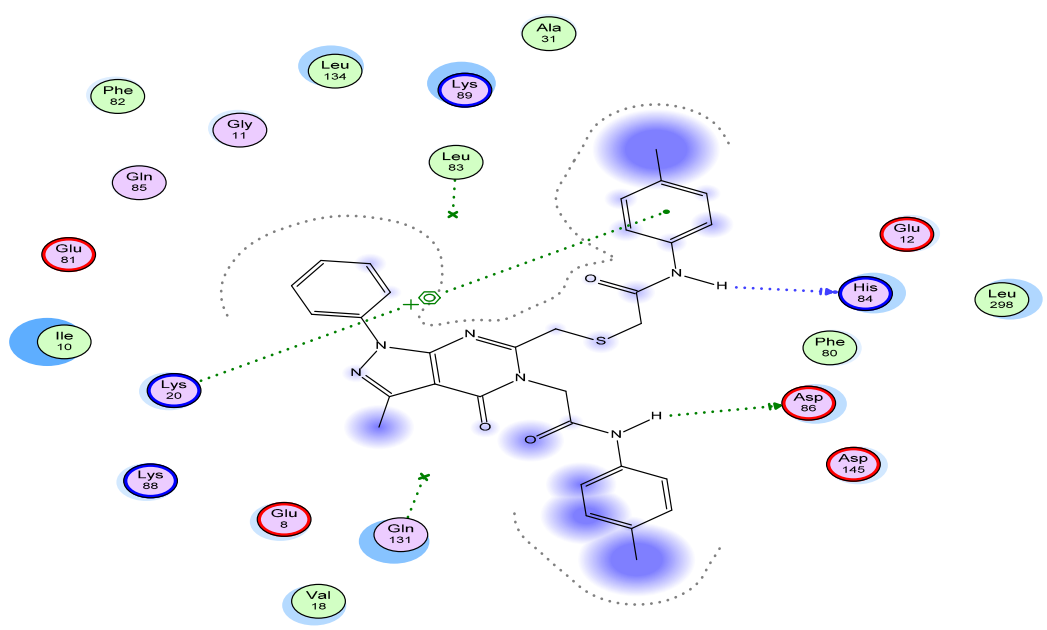

(b)

Figure 7. (a) Proposed binding mode of compound $\mathbf{1 1 b}$ inside the active site of CDK resulting from docking, the most important amino acids are shown together with their respective numbers. Compound $\mathbf{1 1 b}$ forms two H-bonds with Asp 86 and His 84 through its $2 \mathrm{NH}$ groups of the two $\left(\mathrm{SCH}_{2} \mathrm{CONH}\right)$ groups, the other interaction is arene cation interaction with Lys 20 amino acid. (b) 2D interaction of compound 14b with Asp 86 and His 84 amino acids. 

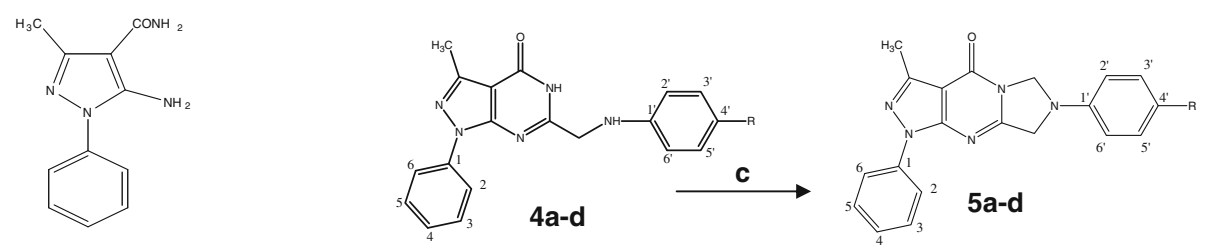

1

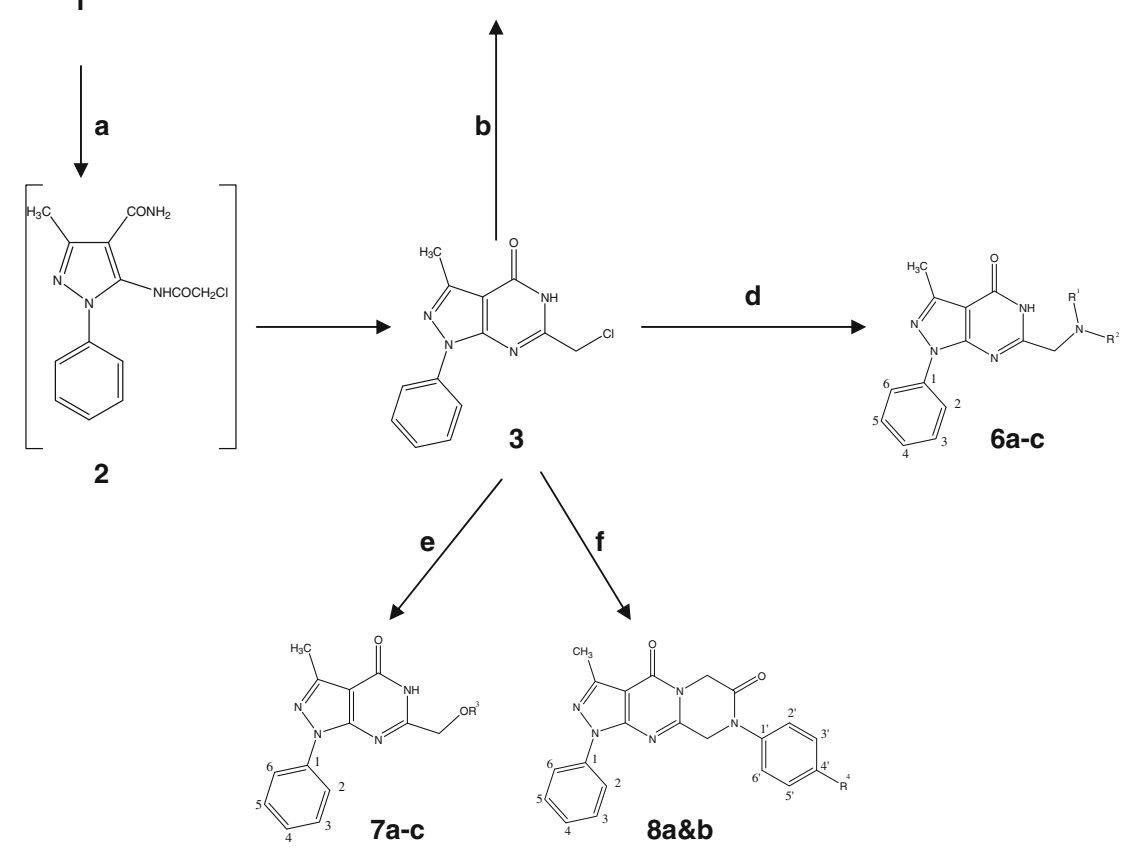

4,5,a, $\mathrm{R}=\mathrm{H}$,

$4,5 \mathrm{~b}, \mathrm{R}=\mathrm{CH}_{3}$,

$4,5 \mathrm{c}, \mathrm{R}=\mathrm{OH}$,

4,5 d, $\mathrm{R}=\mathrm{COOH}$,

6,a, $\mathbf{R}^{1} \mathbf{R}^{2}=\mathrm{N}\left(\mathrm{C}_{2} \mathrm{H}_{5}\right)_{2}$,

6, b, $\mathbf{R}^{1} \mathbf{R}^{2}=\mathrm{N}\left(\mathrm{C}_{5} \mathrm{H}_{10}\right)$

6,c, $\mathbf{R}^{1} \mathbf{R}^{2}=\mathbf{N}\left(\mathrm{C}_{4} \mathrm{H}_{8}\right) \mathrm{O}$,

7, a, $\mathbf{R}^{3}=\mathrm{CH}_{3}$,

$7, b, \mathbf{R}^{3}=\mathrm{C}_{2} \mathrm{H}_{5}$,

7, c, $\mathbf{R}^{3}=\mathrm{C}_{3} \mathbf{H}_{7}$,

$8, \mathbf{a}, \mathbf{R}^{4}=\mathbf{H}$,

$8, b, \mathrm{R}^{4}=\mathrm{CH}_{3}$.

Scheme 1. Reagents: (a) chloroacetyl chloride; (b) primary aliphatic amines; (c) formaldehyde; (d) secondary aliphatic amines; (e) different alkoxides and (f) chloro acetanilide derivatives.

certain compounds and this hydrogen bonding occurred with Gln 8, 131, His 84, Lys 89, 128, 129, Thr 14 and Asp 56, 145 amino acids (figures 3-7). However, most of our compounds interact with the receptor with minimum binding energy scores as shown in table 1 .

\subsection{Experimental section}

Reaction of 5-amino-3-methyl-1-phenyl-1 $H$-pyrazole4-carboxamide (1), ${ }^{28}$ with chloroacetyl chloride yielded 6-chloromethyl-3-methyl-1-phenyl pyrazolo [3, 4- $d]$ pyrimidin-4-one (3), which was used as a starting material for synthesis of novel pyrazolopyrimidines. Cyclization of 1 was preceded through chloroacetylation of amino group forming chloroacetyl aminopyrazole carboxamide $\mathbf{2}$ as non-isolatable intermediate followed by dehydration to afford $\mathbf{3}$. Structure of compound $\mathbf{3}$ was established on the basis of spectral analyses. IR spectrum showed absorption band at 3276 and $75 \mathrm{~cm}^{-1}$ attributed to the presence of $(\mathrm{NH})$ and $(\mathrm{C}=\mathrm{O})$ groups, respectively. Moreover, ${ }^{1} \mathrm{H}-\mathrm{NMR}$ spectrum of compound 3 revealed a single signal at $\delta 4.45$ assigned to the two protons of $\left(\mathrm{CH}_{2}-\mathrm{Cl}\right)$ group, and a singlet at $\delta 12.61, \mathrm{D}_{2} \mathrm{O}$ exchangeable attributed to the $(\mathrm{NH})$ proton. Also, its mass spectrum exhibited a molecular ion peak at $m / z 274$ which agreed with the expected structure.

The key intermediate $\mathbf{3}$ was used as an alkylating agent to be reacted with different primary aliphatic amines, secondary aliphatic amines and some alkoxides 


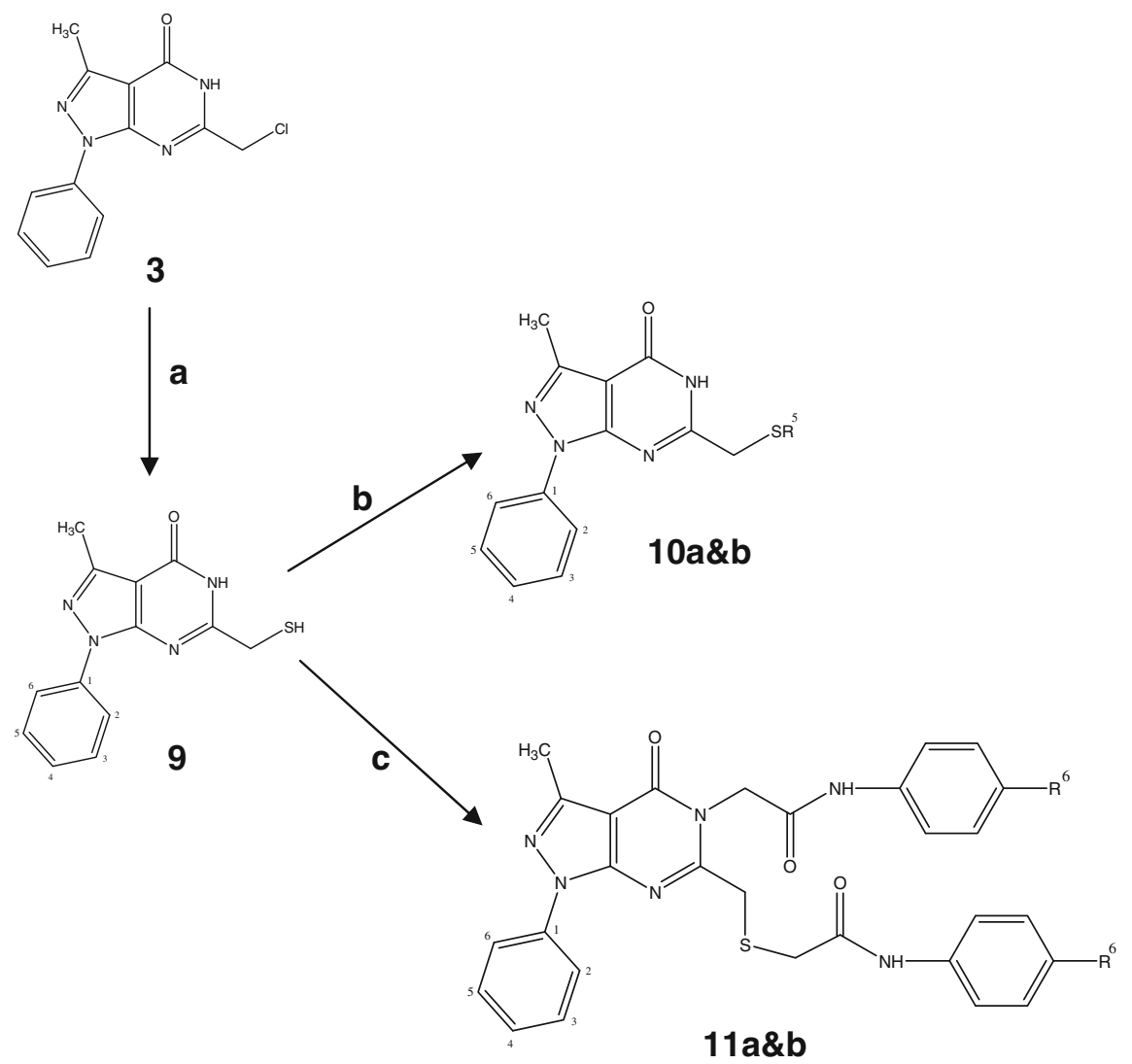

$$
\begin{aligned}
& 10, a, R^{5}=\mathrm{CH}_{2} \mathrm{COOC}_{2} \mathrm{H}_{5}, \\
& 10, \mathrm{~b}, \mathrm{R}^{5}=\mathrm{CH}_{2} \mathrm{C}_{6} \mathrm{H}_{5} \\
& 11, \mathrm{a}, \mathrm{R}^{6}=\mathrm{H}, \\
& 11, \mathrm{~b}, \mathrm{R}^{6}=\mathrm{CH}_{3}
\end{aligned}
$$

Scheme 2. Reagents: a, thiourea; b, a) ethyl chloroacetate, b) benzyl chloride; c, a) anilide, b) $p$-tolyl anilide.

giving series $\mathbf{4 a - d}, \mathbf{6 a}-\mathbf{c}$ and $\mathbf{7 a}-\mathbf{c}$, respectively. All the prepared compounds were confirmed by element analysis and spectral data. Mannich reaction of arylaminomethylpyrazolopyrimidines $\mathbf{4 a - d}$ with formalin $(30 \%)$ solution was preceded via hydroxyl methylation of the $\mathrm{NH}$ group of aryl amino group which spontaneously underwent elimination of water to yield 5ad. Reaction of formaldehyde carbonyl group occurred at the amino $\mathrm{NH}$ rather than the pyrimidine $\mathrm{NH}$ which is present in tautomerism with the adjacent carbonyl group. The structure of 5a-d was confirmed using spectral analyses. IR spectra of compounds 5a-d showed disappearance of bands characteristic of $\mathrm{NH}$ groups in the precursor compound. Also showed absorption bands at the range $1704-1664 \mathrm{~cm}^{-1}$ for $(\mathrm{C}=\mathrm{O})$ group. Their ${ }^{1} \mathrm{H}-\mathrm{NMR}$ showed the disappearance of $\mathrm{D}_{2} \mathrm{O}$ exchangeable signals due to $2 \mathrm{NH}$ protons and appearance of two singlet at $\delta 4.60-4.70$ and at $\delta 5.29-5.37$ characteristic of $2 \mathrm{CH}_{2}$ groups.
Before adding alkyl pharmacophore to $\mathrm{N} 5 \mathrm{H}$ of pyrimidine moiety, compound $\mathbf{3}$ was reacted with chloroacetanilide derivatives which resulted in the tricyclic products $\mathbf{8 a}$ and $\mathbf{b}$. This reaction was suggested to be proceeded via double alkylation; one of them is of the amidic $\mathrm{NH}$ with methylene chloride group of pyrimidine $\mathbf{3}$ and the other for $\mathrm{N} 5 \mathrm{H}$ of pyrimidine $\mathbf{3}$ and $-\mathrm{CH}_{2} \mathrm{Cl}$ of the anilide. Structure of compounds $8 \mathbf{a}$ and b was confirmed by its IR, which revealed two $(\mathrm{C}=\mathrm{O})$ groups at the range of $1705-1704 \mathrm{~cm}^{-1}$ and disappearance of NH group. ${ }^{1} \mathrm{H}-\mathrm{NMR}$ spectra showed two singlet signals at $\delta 4.66-4.77$ and at $\delta 5.01-5.34$ characteristic of $2 \mathrm{CH}_{2}$ groups. Also, mass spectrum of compounds 8 $\mathbf{a}$ and $\mathbf{b}$ exhibited a molecular ion peak at $m / z 371$ and $m / z 385$, respectively (scheme 1 ).

On the other hand, 6-chloromethyl-3-methyl-1phenyl pyrazolo $[3,4-d]$ pyrimidin-4-one (3) was converted into the corresponding mercapto derivative 9 by refluxing with thiourea in ethanol resulting in the 
formation of the isothiouronium salt which was then treated with sodium hydroxide followed by acidification with hydrochloric acid to give compound 9. Structure of compound 9 was elucidated from its element and spectral analyses. IR spectrum showed absorption band at $2733 \mathrm{~cm}^{-1}$ for SH group. Its $1 \mathrm{H}-\mathrm{NMR}$ showed a single signal at $\delta 3.97$ for $-\mathrm{CH}_{2}$. Mass spectrum of compound 9 revealed a peak at $m / z 272$ corresponding to molecular ion peak.

As an extension of this synthetic route, compound 9 was alkylated using halogenated compounds such as ethyl chloroacetate and/or benzyl chloride to give S-alkylated compounds 10a and b. Structure of 10a and $\mathbf{b}$ was elucidated using element and spectral analyses. IR spectra showed disappearance of SH peak and appearance of absorption bands in the range of $3445-3439 \mathrm{~cm}^{-1}$ for $(\mathrm{NH})$ group, $1674-1687 \mathrm{~cm}^{-1}$ for $(\mathrm{C}=\mathrm{O})$, and $1734 \mathrm{~cm}^{-1}$ for $(\mathrm{C}=\mathrm{O})$ of ester moiety in compound 10a. The ${ }^{1} \mathrm{H}-\mathrm{NMR}$ showed the appearance of new signals characteristic for $\left(-\mathrm{S}-\mathrm{CH}_{2}-\right)$ at $\delta$ 3.73, 3.97, also, mass spectra for compounds 10a and b showed molecular ion peaks at $\mathrm{m} / \mathrm{z} 358$ and 362 respectively which is in agreement with the suggested structure.

In addition to S-alkylation, chloroacetanilide derivatives were used for S-alkylation, while the resulting structures 11a and $\mathbf{b}$ were the doubly alkylated products on $\mathrm{S}-$ and $\mathrm{N}-$ atoms. This was attributed to the effect of $(-\mathrm{CONH})$ group of the chloroacetanilide derivatives which increased the positive charge at $\left(\mathrm{CH}_{2}\right)$ and facilitated the acceptance of lone pairs on $\mathrm{S}$ and $\mathrm{N}$ atoms leading to alkylation on both $\mathrm{S}$ and $\mathrm{N}$. The structures were confirmed by micro analytical and spectral data, IR spectra showed absorption bands in the range of 3437-3289 $\mathrm{cm}^{-1}$ for two amidic NH groups, 1691$1662 \mathrm{~cm}^{-1}$ for $(\mathrm{C}=\mathrm{O})$ of pyrimidine ring and $(2 \mathrm{C}=\mathrm{O})$ of amidic bond. ${ }^{1} \mathrm{H}$ - NMR showed the appearance of single signals characteristic for $\left(-\mathrm{CH}_{2} \mathrm{~S}-\right)$ at $\delta 3.49$, 3.54 , singlet signals at $\delta 4.08,4.09$ for $\left(-\mathrm{S}-\mathrm{CH}_{2}-\right)$, at $\delta$ 5.05-5.08 for $\left(-\mathrm{N}-\mathrm{CH}_{2}-\mathrm{CO}-\right)$, also appearance of two $\mathrm{D}_{2} \mathrm{O}$ exchangeable singlet signals at $\delta$ 9.86-9.94 and 10.36-10.45 referred to the two $(\mathrm{NH})$ groups. Mass spectra for compounds 11a and $\mathbf{b}$ showed molecular ion peak at $m / z 538$ and 566, respectively which confirmed the suggested structure (scheme 2).

\subsection{Antitumour activity}

Olomoucine I was used as the reference drug in this study. Some of the synthesized compounds were selected and screened for their anticancer activity. Each compound was tested at different concentrations against human breast cancer cell line. The relationship between survival fraction and drug concentration was plotted to obtain the survival curve of human breast cancer cell line (MCF-7). The response parameters calculated

Table 2. In vitro cytotoxic activity of some newly synthesized compounds and the standard olomoucine I.

\begin{tabular}{|c|c|c|c|c|c|c|c|}
\hline \multirow[b]{3}{*}{ Compound } & \multicolumn{7}{|c|}{ Survival fraction $(\%)$} \\
\hline & \multicolumn{7}{|c|}{ Concentration $(\mu \mathrm{g} / \mathrm{mL})$} \\
\hline & 50 & 25 & 12.5 & 5 & 2.5 & $\mathrm{IC}_{50}^{\mathrm{a}}(\mu \mathrm{g} / \mathrm{ml})$ & $\mathrm{IC}_{50}(\mu \mathrm{M})$ \\
\hline $4 b$ & 0.135 & 0.132 & 0.101 & 0.111 & 0.149 & 2.7 & 0.008 \\
\hline $4 d$ & 0.163 & 0.105 & 0.473 & 0.918 & 0.987 & 11.6 & 0.030 \\
\hline $5 \mathbf{a}$ & 0.124 & 0.233 & 0.405 & 0.652 & 0.804 & 9.6 & 0.027 \\
\hline $5 c$ & 0.289 & 0.201 & 0.271 & 0.472 & 0.631 & 8.84 & 0.013 \\
\hline $5 d$ & 0.113 & 0.371 & 0.583 & 0.725 & 0.901 & 17.6 & 0.045 \\
\hline $\mathbf{6 a}$ & 0.188 & 0.181 & 0.297 & 0.523 & 0.634 & 5.55 & 0.017 \\
\hline $\mathbf{6 b}$ & 0.255 & 0.139 & 0.152 & 0.418 & 0.620 & 4.2 & 0.012 \\
\hline 6c & 0.173 & 0.391 & 0.581 & 0.762 & 0.940 & 17.7 & 0.054 \\
\hline $7 \mathbf{a}$ & 0.233 & 0.140 & 0.239 & 0.521 & 0.761 & 5.85 & 0.021 \\
\hline $7 \mathbf{b}$ & 0.214 & 0.297 & 0.392 & 0.754 & 0.939 & 10.2 & 0.035 \\
\hline $7 c$ & 0.133 & 0.235 & 0.515 & 0.727 & 0.913 & 13.2 & 0.044 \\
\hline $8 \mathbf{a}$ & 0.201 & 0.113 & 0.186 & 0.307 & 0.524 & 3.45 & 0.009 \\
\hline $8 b$ & 0.198 & 0.080 & 0.154 & 0.614 & 0.810 & 6.6 & 0.080 \\
\hline $10 \mathbf{a}$ & 0.234 & 0.161 & 0.377 & 0.599 & 0.771 & 8.4 & 0.023 \\
\hline $10 b$ & 0.048 & 0.076 & 0.086 & 0.103 & 0.311 & 2.85 & 0.007 \\
\hline $11 a$ & 0.123 & 0.077 & 0.080 & 0.404 & 0.612 & 4.2 & 0.007 \\
\hline 11b & 0.236 & 0.209 & 0.129 & 0.202 & 0.483 & 2.85 & 0.004 \\
\hline Olomoucine & - & - & - & - & - & - & 7 \\
\hline
\end{tabular}

${ }^{\text {a }}$ The values given are means of three expirements

Bold numbers refer to the most potent compounds (lower IC50) relative to the standard 


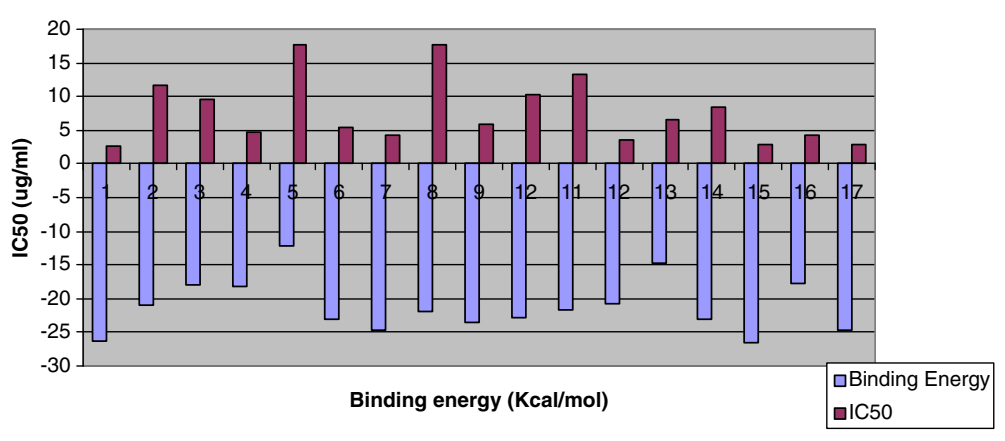

Chart 1. Docking scores against $\mathrm{IC}_{50}$.

was the $\mathrm{IC}_{50}$ value, which corresponds to the compound concentration causing $50 \%$ mortality in net cells (table 2).

\section{Conclusions}

The present data showed that compounds $\mathbf{4 b}, \mathbf{8 a}, \mathbf{1 0 b}$, 11a and $\mathbf{b}$ exhibited promising in vitro cytotoxic activity against (MCF-7) giving the highest cytotoxic activity between 0.009 and $0.004 \mu \mathrm{M}$ when compared to the other test compounds and olomoucine $\mathbf{I}(\mathbf{7} \mu \mathrm{M})$ as a reference drug. All the other test compounds showed higher $\mathrm{IC}_{50}$ values than that of the reference drug, ranging from 0.012 to $0.080 \mu \mathrm{M}$. Docking studies confirmed this result as compounds $4 \mathbf{b}, 8 \mathbf{a}, 10 \mathbf{b}, 11 \mathbf{a}$ and b showed low binding energy scores from -24.2298 to $-16.2786 \mathrm{Kcal} / \mathrm{mol}$ ), also showed binding mode similar to olomoucine I which was reported to possess CDK inhibitory effects. $\mathrm{IC}_{50}$ values were plotted against binding energy scores which revealed that there was some sort of consistency between the docking studies and in vitro screening (chart 1).

\section{References}

1. Yenugonda V M, Deb T B, Grindrod S C, Dakshanamurthy S, Yang Y, Paige M and Brown M L 2011 Bioorg. Med. Chem. 192714

2. Otyepka M, Krystof V, Havlicek L, Siglerova V, Strnda M and Koca J 2000 J. Med. Chem. 432506

3. Cavasotto C N, Ortiz M A, Abagyan R A and Piedrafita F J 2006 Bioorg. Med. Chem. Lett. 161969

4. Peat A J, Garrido D, Boucheron J A, Schweiker S L, Dickerson S H, Wilson J R, Wang T Y and Thomson S A 2004 Bioorg. Med. Chem. Lett. 142127

5. Peat A J, Boucheron J A, Dickerson S H, Garrido D, Mills W, Peckham J, Preugschat F, Smalley T, Schweiker S L, Wilson J R, Wang T Y, Zhou H Q and Thomson S A 2004 Bioorg. Med. Chem. Lett. 142121

6. Gupta S, Rodrigues L M, Esteves A P, Oliveira-Campos A M F, Nascimento M S J, Nazareth N, Cidade H, Neves M P, Fernandes E, Pinto M, Cerqueira N M F S A and Bras N 2008 Eur. J. Med. Chem. 43771
7. Huang $\mathrm{X}$, Finerty $\mathrm{P} \mathrm{Jr}$, walker $\mathrm{J}$ R, Butler-Cole C, Vedadi M, Schapira M, Parker S A, Turk B E, Thompson D A and Dhe-Paganon S 2009 J. Struct. Biol. 16588

8. Schenone S, Zanoli S, Brullo C, Crespan E and Maga G 2008 Curr. Drug Therapy 3158

9. Huang H, Ma J, Shi J, Meng L, Jiang H, Ding J and Liu H 2010 Bioorg. Med. Chem. 184615

10. Tintori C, Magnani M, Schenone S and Botta M 2009 Eur. J. Med. Chem. 44990

11. Kumar A, Ahmad I, Chhikara B S, Tiwari R, Mandal D and Parang K 2011 Bioorg. Med. Chem. Lett. 211342

12. Baraldi P G, El-Kashef H, Farghaly A R, Vanelle P and Fruttarolo F 2004 Tetrahedron 605093

13. Salaheldin A M, Oliveira-Campos A M F and Rodrigues L M 2009 Synth. Commun. 391186

14. Park K S, Kim J, Chong Y and Choo H 2007 Bull. Korean Chem. Soc. 28211

15. Ibrahim D A, El-Metwally A M and Al-Arab E E 2009 Arkivoc vii 12

16. Patrick G L 2008 An introduction to medicinal chemistry 4th edn. (England: Oxford) p. 519

17. Abunada N M, Hassaneen $\mathrm{H}$ M, Kandile N G and Miqdad O A 2008 Molecules 131501

18. Dave C G and Shah R D 2002 Molecules 7554

19. Ismail Z H, Abdel-Gawad S M, Abdel-Aziem A and Ghorab M M 2003 Phosphorus Sulfur Silicon 1781795

20. Rashad A E, Hegab M I, Abdel-Megeid R E, Fathalla N and Abdel-Megeid F M E 2009 Eur. J. Med. Chem. XXX 1

21. Rashad A E, Hegab M I, Abdel-Megeid R E, Micky J A and Abdel-Megeid F M E 2008 Bioorg. Med. Chem. 167102

22. Schenone S, Bruno O, Bondavalli F, Ranise A, Mosti L, Menozzi G, Fossa P, Donnini S, Santoro A, Ziche M, Manetti F and Botta M 2004 Eur. J. Med. Chem. 39939

23. Moukha-Chafiq O and Taha M L 2006 Nucleos. Nucleot. Nucl. Acids 25849

24. Ghorab M M, Ragab F A, Alqasoumi S I, Alafeefy A M and Aboulmagd S A 2010 Eur. J. Med. Chem. 45171

25. Schenone S, Bruno O, Radi M and Botta M 2009 Minirev. Org. Chem. 6220

26. Website: http://www.rcsb.org/pdb

27. Warren J, Bokesch H, Kenney S and Boyed M R 1990 J. Nat. Cancer Inst. 821107

28. Marsico J et al. 1973 United States Patent, 3,760,084, 18 September 